\title{
A grain-scale model for high-cycle fatigue degradation in polycrystalline materials
}

\author{
Ivano Benedetti ${ }^{\mathrm{a}, *}$, Vincenzo Gulizzi ${ }^{\mathrm{a}}$ \\ ${ }^{a}$ Department of Civil, Environmental, Aerospace, and Materials Engineering - DICAM, \\ Università degli Studi di Palermo, Viale delle Scienze, Edificio 8, \\ Palermo, 90128, Italy.
}

\begin{abstract}
A grain-scale three-dimensional model for the analysis of fatigue intergranular degradation in polycrystalline materials is presented. The material microstructure is explicitly represented through Voronoi tessellations, of either convex or non-convex domains, and the mechanics of individual grains is modelled using a boundary integral formulation. The intergranular interfaces degrade under the action of cyclic loads and their behaviour is represented employing a cohesive zone model embodying a local irreversible damage parameter that evolves according to high-cycle continuum damage laws. The model is based on the use of a damage decomposition into static and cyclic contributions, an envelope load representation and a cycle jump strategy. The consistence between the cyclic damage and the action of the external loads, which contribute to the damage due to the redistribution of intergranular tractions between subsequent cycle jumps, is assessed at each solution step, so to capture the onset of macro-failure when the external actions cannot be equilibrated anymore by the critically damaged interfaces. Several numerical tests are reported to illustrate the potential of the developed method, which may find application in multiscale modelling of fatigue material degradation as well as in the design of micro-electro-mechanical devices (MEMS).
\end{abstract}

Keywords: High-cycle fatigue, Polycrystalline materials, Micro-mechanics, Intergranular degradation, Boundary element method

\footnotetext{
${ }^{*}$ Corresponding author

Email address: ivano.benedetti@unipa.it (Ivano Benedetti)
} 


\section{Introduction}

Materials fatigue degradation is a common cause of structural failure in several applications in the automotive, aerospace, maritime and oil industries [1 2, 3, 4, 5]. The design of engineering components subjected to cyclic loads has been traditionally based on phenomenological semi-empirical tools, such as the Whöler $\sigma-N$ curves [6], Basquin's power laws [7] or Goodman-type diagrams [8]. More recently, the analysis of fatigue induced cracks initiation and propagation has been addressed employing continuum damage mechanics [9] or fracture mechanics [10, 11, 4] approaches, within the framework of safe-life, fail-safe or damage-tolerance design methodologies.

The above tools have been and are currently used in the analysis and design of macroscopic mechanical components subjected to cyclic loads. However, the last few decades have seen a surge of interest in the study and modelling of materials at the micro/nano scales [12, 13, 14]. Remarkable interest has been attracted by investigations at the meso-scale, with the explicit representation of the heterogeneous constituents of materials often assumed as homogeneous at the macro-scale; outstanding examples are provided either by the micro-mechanical studies on fibre-reinforced composites [15, 16 17], with the explicit representation of individual laminae or even fibres, matrix and related interfaces, or by the analysis of polycrystalline aggregates, with the direct account of individual grains and intergranular interfaces [18, 13].

The trend towards computational micro-mechanics has different motivations. From a macroscopic point of view, it is driven by the scientific interest for better understanding of materials behaviour, beyond their phenomenological representation, and the consequential technological drive towards the improvement of materials performances by chemical pathways, as for example in the case of grain-boundary engineering [19], or by topological/morphological control of the microstructure, as in the manufacturing of foam and lattice materials [20 21]. From an inherently microscopic point of view, such interest is often related to the rapid development and widespread use of micro-electro-mechanical systems (MEMS), today routinely employed and well established in several engineering applications as transducers, gears and mechanical 
linkages [22]. In any case, the rapid development of computational micro-mechanics has certainly benefitted from the development of experimental techniques for materials characterisation [23, 24] and from the current affordability of high performance computing (HPC) [25].

In the sketched background, the study of materials damage and failure micro35 mechanisms has assumed particular importance, as it allows the enhanced understanding of damage nucleation across length scales in engineering applications [26] and it may find direct application in the design of MEMS [27, 28, 29, 30]. In this study, we focus on modelling of degradation and failure in polycrystalline aggregates subjected to cyclic loads. Polycrystalline materials, either metals or ceramics, are widely employed in a diverse range of engineering applications. Polycrystalline silicon, or polysilicon, is routinely used for the production of MEMS components, which are often subjected to cyclic loads [31, 32, 33].

The microstructure of polycrystalline materials is characterised by the shape and crystallographic orientation of the grains and by the physical and chemical prop${ }_{45}$ erties of the intergranular interfaces, which play an important role in their micromechanics. Several computational techniques have been developed for the analysis of polycrystalline materials at the micro-scale and for the study of their mechanical, damage and failure behaviour [34 35]. In particular, grain-scale models have been developed for material homogenisation [36, 37], quasi-static failure [38, 39] and stress 50 corrosion cracking [40, 41, 42, 43]. Also the progressive fatigue degradation of polycrystalline aggregates subjected to cyclic loads has been addressed in the literature. Manonukul and Dunne [44] and Cheong et al. [45] have developed crystal plasticity finite element frameworks with fatigue crack initiation criteria based on the accumulation of slip to quantify fatigue damage and crack initiation in low and/or high-cycle fatigue of polycrystalline aggregates. A review of the grain-scale, or microstructuresensitive, computational models for fatigue in polycrystalline or poly-phase materials can be found e.g. in Refs. [13, 46]. Several of such studies are often devoted to microstructures in which the accumulation of plastic slip is the dominant mechanism triggering the initiation of fatigue cracks at the grain scale. On the other hand, Jalalah60 madi et al. [28] have developed a Voronoi finite element model (VFEM) for the analysis 
of fatigue damage and failure in MEMS; they employed a stress reversal based quasibrittle damage evolution law suitable for fine-grain metal microstructures with highly confined plasticity, to model intergranular crack initiation and propagation stages and used it to investigate the effects of microstructure randomness on the fatigue of ${ }_{65}$ MEMS. The approach was extended to three-dimensional aggregates by Bomidi et al. [47].

In this work, a three-dimensional (3D) grain-scale boundary integral formulation for the analysis of polycrystalline aggregates under cyclic loading is proposed. Only intergranular fatigue degradation is considered here: the formulation is then suitable for aggregates/conditions in which plastic slip is absent or highly confined and then negligible [48, 49, 50]. Such conditions are often met in fine-grain MEMS applications [47]. The method is based on a grain-boundary integral approach previously developed and applied for computational material homogenisation [51 52], quasi-static intergranular and transgranular degradation and failure [53, 54, 55, 56, 57, 58, 59],

75 hydrogen assisted stress corrosion cracking [60] and multiscale analysis of damage initiation, evolution, coalescence and fracture of polycrystalline components [61 62]. The formulation is based on a boundary integral representation of the mechanics of the individual grains, coupled with a cohesive zone model of the progressive degradation of the intergranular interfaces. In this work, to model fatigue degradation, the 80 intergranular damage is decomposed into a contribution induced by the cyclic loads and a contribution from the quasi-static action of the applied loads, as it is often done in high-cycle fatigue analysis. This damage decomposition is used in conjunction with an envelope load representation of the external cyclic actions and a cycle jump strategy that, due to the practical unfeasibility of cycle-by-cycle analysis of high-cycle ${ }_{85}$ problems, is adopted to render the computation amenable. The formulation offers the advantage of expressing the micro-mechanical problem in terms of intergranular displacements and tractions only, which are then directly used in the expression of the traction-separation laws. Moreover, since only the discretisation of the grain boundaries is required, the methodology offers a simplification of the data prepa90 ration stage and a reduction of the number of degrees of freedom required by the micro-mechanical analysis, with resulting computational benefits. 
The paper is organised as follows. The details about the generation of the analysed artificial polycrystalline morphologies are given in Section 2 where also a strategy for the generation of non-convex aggregates is described. The grain-boundary formulation is described in Section 3 where the employed boundary integral equations, the interface laws embodying fatigue degradation laws and the system solution are addressed. In particular, the description of the strategy used to model the intergranular degradation is described in Section 3.3 . where the damage evolution decomposition is described and discussed on the background of the approaches used in the literature to address fatigue degradation; the envelope load approach and the cycle jump strategy are discussed in Section 3.4 Section 4 reports on the numerical tests performed using the developed method. Some discussion about the obtained results and an outline of possible further developments is given in Section 5 before the Conclusions.

\section{Generation and meshing of artificial crystal aggregates}

The first essential item of the proposed framework is a suitable artificial representation of polycrystalline microstructures. In this study, the fatigue behaviour of crystal aggregates contained within the volume $\mathscr{V}$, bounded by the surface $\mathscr{B}=\partial \mathscr{V}$, is considered. The polycrystalline aggregates are modelled employing a multi-region boundary element formulation [63], in which each different region represents an individual grain, with its own specific crystallographic properties and orientation in space.

Several different techniques have been used in the literature to represent polycrystalline aggregates, with resulting representations varying from the most schematic, see e.g. Ref. [64], to the most realistic, see e.g. Ref.[65] where the microstructure representation is based on 3D X-ray diffraction contrast tomography. In this work, the analysed morphologies are generated through Voronoi [37, 66] or more general Laguerre [67, 68, 69 tessellations of the analysed domain $\mathscr{V}$. Such algorithms are relatively simple, yet mathematically well defined, space filling geometric subdivisions of the considered volume that provide suitable first-order approximations of 
ual grain $g$, is a convex polyhedron with the boundary $S^{g}$ comprised of the union of flat convex polygonal faces $F_{n}^{g}$ with $n=1, \ldots, N_{f}^{g}$ and $N_{f}^{g}$ denoting the number of faces of $g$. The tessellations can be practically built using open source software packages such as Voro++ (http://math.1bl.gov/voro++/) [70] or Neper (http://neper. sourceforge.net) [66]. In this study, Neper has been employed; however, what follows can be applied also if other software packages are adopted for generating the domain tessellations.

In several studies, generally concerned with material homogenisation, the considered polycrystalline domains $\mathscr{V}$ have typically cubic or prismatic shape and contain a certain number of grains that are subsequently analysed with the aim of identifying some representative volume elements (RVEs), see Fig.(1). Cubic or prismatic boxes, and in general convex domains, can be straightforwardly generated with available software packages, which often provide methods for cutting grains or aggregates. However, the cutting operation, by its own nature, is not generally suitable for the generation of non-convex domain. With the aim of considering either convex or nonconvex polycrystalline components, we have developed a dedicated technique for the tessellation of non-convex domains, similar to that used in Ref.[47]. The method is described next.

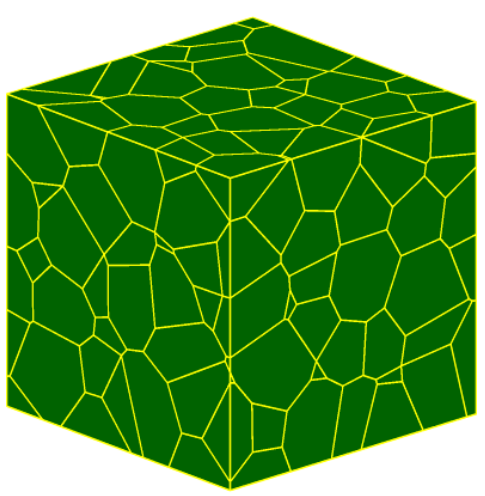

Figure 1: Tessellations of cubic or prismatic domains are often used in homogenisation studies to identify representative volume elements for polycrystalline materials. 
The non-convex domain $\mathscr{V}$, bounded by the surface $\mathscr{B}=\partial \mathscr{V}$, is considered; $\mathscr{V}$ can be enclosed within the parallelepiped bounding box $\mathscr{C}$. The tessellation of $\mathscr{V}$ is articulated in the following different stages, as illustrated in Fig.(2). a) Seeds generation: initially, a certain number of seeds, with their associated weights if Laguerre tessellations are employed, is scattered within the box $\mathscr{C}$, so to achieve the target grains density. In the generation, for reducing the number of small geometrical entities, namely short edges and small faces, which would induce subsequent excessive mesh refinements, an additional hardcore condition is enforced as in Ref.[55]; b) Seeds selection: the seeds within the boundary surface $\mathscr{B}$, which can have an implicit or parametric representation, are selected, while the seeds within $\mathscr{C}$ but outside $\mathscr{B}$ are removed; c) Seeds mirroring: the surviving seeds closer to the surface $\mathscr{B}$ are mirrored with respect to $\mathscr{B}$ itself. Specifically, the distance between the generic point $P$ and $\mathscr{B}$ is computed, then identifying the projection point $Q \in \mathscr{B}$; the mirrored point $P^{\prime}$ is then generated as $P^{\prime}=P+2 P Q$. The mirroring process creates outside $\mathscr{B}$, but still within $\mathscr{C}$, a new set of points that have the purpose of inducing, by the very definition of Voronoi tessellation, a set of intergranular interfaces whose envelope will mimic the surface $\mathscr{B}$ itself. Particular care must be used during this stage in proximity of regions of $\mathscr{B}$ with high curvatures. d) Volume tessellation: the bounding box $\mathscr{C}$, which contains $\mathscr{V}$, is tessellated using the available generators, using both the seeds inside and outside $\mathscr{B}$. The available software packages are suitable for performing the tessellation of $\mathscr{C}$, due to its convex prismatic shape; however, as mentioned, the specific 160 placement of the mirrored seeds will induce interfaces tangent to $\mathscr{B}$ itself, see Fig.2c]; e) Grain removal: as the tessellation is completed, there will be grains within $\mathscr{C}$ lying almost entirely outside $\mathscr{B}$; to complete the generation of the intended non-convex polycrystalline domain such grains must then be removed.

Once a polycrystalline morphology of $\mathscr{V}$ is obtained, a suitable mesh must be generated, to discretise the micro-mechanics equations. A specific feature of the proposed formulation is that only the boundary of the grains, and not their interior volume, must be meshed, which induces a reduction in the order of the solving system. This aspect, in conjunction with the fact that the grains faces $F_{n}^{g}$ are a collection of convex flat polygons, allows a remarkable simplification of the data preparation for 


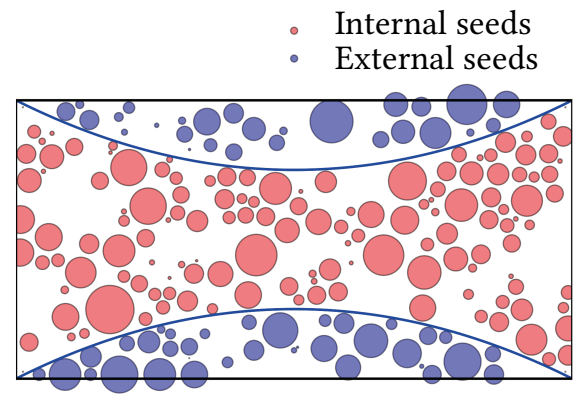

(a)

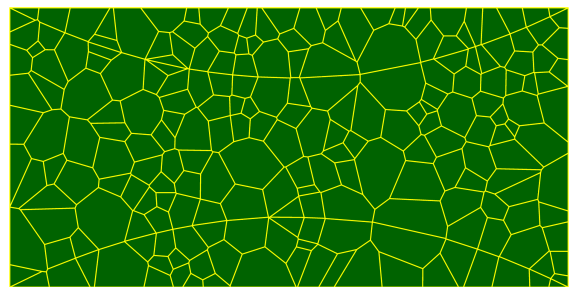

(c)

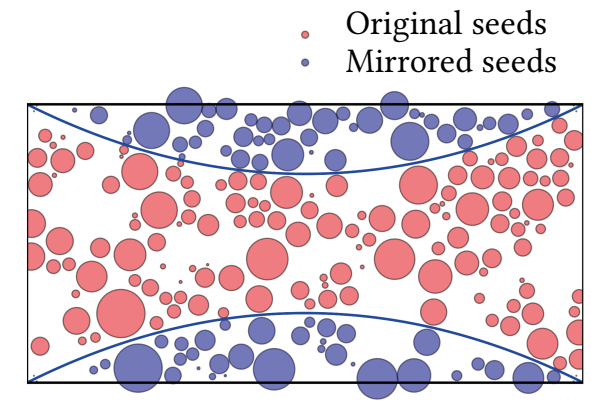

(b)

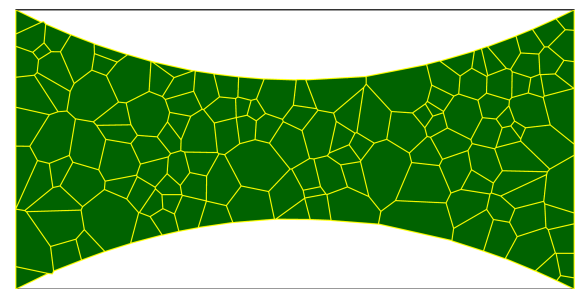

(d)

Figure 2: Schematic representation of the procedure employed for the generation of the tessellation of nonconvex domains: a) Seeds generation and selection; $b$ ) Seeds mirroring with respect to the non-convex domain walls; $c$ ) Tessellation; $d$ ) Grain removal and resultant polycrystalline morphology. 
the computational treatment of the problem. The meshing is performed following the procedure proposed in Ref.[55]: each polygonal face $F_{n}^{g}$ is subdivided into a collection of non-overlapping triangular and quadrangular, continuous and semi-discontinuous elements. The use of semi-discontinuous elements in proximity of the edges of the grains is motivated by the will of avoiding the complexities induced by the collocation 175 of nodes on regions with a non unique definition of the surface normal vector, which would require special treatment within the framework of boundary element formulations, see e.g. [71 72]. The mesh size is selected so that the average element length $l_{e}$ is considerably smaller than the cohesive zone size $L_{c z}$, which can be estimated in terms of the material fracture toughness and the interface strength [73, 74, 75, 53, 54].

180 3. The polycrystalline grain-boundary formulation

In this Section, the grain boundary formulation developed for high-cycle fatigue in polycrystalline aggregates is presented and described. As mentioned above, only intergranular degradation is considered. The polycrystalline aggregate is modelled using a multi-region boundary element formulation [63 76, 51] in which each grain 185 is represented as a 3D generally anisotropic linear elastic crystal, with its own crystallographic features and space orientation, while the intergranular interfaces are modelled as non-linear cohesive regions [54,55,57] that, under the action of cyclic loading, can witness the nucleation, development and coalescence of micro-damage up to the evolution into frictional micro-cracks. This modelling strategy is suitable for aggregates and/or operational conditions in which the material evolution is mainly localised at the intergranular interfaces, while it is assumed that the grains interior does not undergo significant deformation, degradation or phase changes. A similar approach has been proposed in Ref.[47] for polysilicon aggregates with highly confined plasticity.

In the presentation that follows, the final compact general form of the solving system for the overall aggregate is given first. The specific items entering the formulation, namely the boundary element matrix terms related to the bulk grains, the cohesive laws related to the intergranular interfaces and the fatigue degradation laws, 
are described in the subsequent sections, so that the readers familiar with a specific item can focus on the details of interest with an idea of their collocation within the overall framework.

\subsection{Grain-boundary polycrystalline system}

Considering an artificial aggregate consisting of $N_{g}$ grains contained within the volume $\mathscr{V}$ bounded by the surface $\mathscr{B}$, after the meshing and discretisation process and application of boundary and intergranular interface conditions, the general compact form of the system of equations for the polycrystalline aggregate reads

$$
\left[\begin{array}{c}
\boldsymbol{A} \cdot \boldsymbol{X} \\
\boldsymbol{I}\left[\boldsymbol{X}, \boldsymbol{d}^{*}(N)\right]
\end{array}\right]=\left\{\begin{array}{c}
\boldsymbol{C} \cdot \boldsymbol{Y}(\lambda) \\
\mathbf{0}
\end{array}\right\},
$$

with

$$
\boldsymbol{A}=\left[\begin{array}{cccc}
\boldsymbol{A}^{1} & \mathbf{0} & \cdots & \mathbf{0} \\
\mathbf{0} & \boldsymbol{A}^{2} & \cdots & \mathbf{0} \\
\vdots & \vdots & \ddots & \vdots \\
\mathbf{0} & \mathbf{0} & \cdots & \boldsymbol{A}^{N_{g}}
\end{array}\right], \quad \boldsymbol{C}=\left[\begin{array}{cccc}
\boldsymbol{C}^{1} & \mathbf{0} & \cdots & \mathbf{0} \\
\mathbf{0} & C^{2} & \cdots & \mathbf{0} \\
\vdots & \vdots & \ddots & \vdots \\
\mathbf{0} & \mathbf{0} & \cdots & C^{N_{g}}
\end{array}\right]
$$

In Eq.(1) the vector $\boldsymbol{X}$ contains the unknown components of grain-boundary nodal displacements $u_{i}$ and tractions $t_{i}$, which are the primary variables of the formulations;

205 by contrast, $\boldsymbol{Y}(\lambda)$ contains the known components of nodal displacements and tractions, which depend on the load factor $\lambda$. In Eq.(2), the matrix blocks $\boldsymbol{A}^{k}$ and $\boldsymbol{C}^{k}$, associated with the $k$-th grain, stem from the matrices produced by the application of the boundary element method to the grain $k$, with $A^{k}$ containing columns associated with the unknown degrees of freedom and $\boldsymbol{C}^{k}$ collecting columns corresponding to the known displacement and traction components. The matrix block $\boldsymbol{I}\left[\boldsymbol{X}, \boldsymbol{d}^{*}(N)\right]$ implements the interface equations, where $\boldsymbol{d}^{*}$ is the vector collecting components $d_{k}^{*}$ expressing the local total interface degradation at the generic interface node pair $k$ and $N$ is the parameter counting the number of loading cycles.

For the solution of the polycrystalline high-cycle fatigue problem, Eq.(1) must be suitably used, as discussed in Section 3.4 in the framework of a strategy comprised of several ingredients, namely: $i$ ) a damage decomposition into quasi-static damage $d_{s}^{*}$ 
and cyclic damage $d_{c}^{*}$ contributions, Section 3.3.1 ii) an envelope load representation of the applied cyclic loads; iii) a cycle jump strategy for the actual simulation of highcycle fatigue problems.

Key items of the formulation are then the rules for the evolution of the quasi-static damage $d_{s}^{*}$ and those for evolution of the cyclic damage $d_{c}^{*}$. As it will be described in Section 3.3 .2 the solution of Eq.11 will provide the necessary information for the update of the quasi-static contribution, while the description of the cyclic degradation will require the introduction of additional suitable laws. In this work, as discussed in Section 3.3.3 it is assumed that intergranular interfaces degrade, under cyclic loading, according to a generic law of the form

$$
\frac{\partial \boldsymbol{d}^{*}}{\partial N}=\boldsymbol{\Psi}\left(\boldsymbol{X}, \llbracket \boldsymbol{X} \rrbracket, \boldsymbol{d}^{*}, \boldsymbol{\pi}\right),
$$

where the operator $\llbracket \cdot \rrbracket$ returns the range of a quantity over a load cycle and $\pi$ expresses the dependence on generic material parameters.

Before entering into the details of interfaces modelling strategy, the origin of the terms entering Eq.(2) is considered in Section 3.2

\subsection{Bulk grains modelling}

The matrix blocks $\boldsymbol{A}^{g}$ and $\boldsymbol{C}^{g}$, with $g=1, \ldots, N_{g}$, appearing in Eq.(1.2) stem, as mentioned above, by the boundary element processing of the generic grain $g$ occupying the volume $\mathscr{V}_{g}$ bounded by the surface

$$
\mathscr{B}_{g}=\partial \mathscr{V}_{g}=\bigcup_{n=1}^{N_{f}^{g}} F_{n}^{g},
$$

where $F_{n}^{g}$ is the generic $n$-th face of the grain $g$. The key item of the formulation is the integral representation of the grain boundary displacement field [76 63],

$$
\widetilde{c}_{i j}^{g}(\boldsymbol{\xi}) \widetilde{u}_{j}^{g}(\boldsymbol{\xi})+\sum_{n=1}^{N_{f}^{g}} f_{F_{n}^{g}} \widetilde{T}_{i j}^{g}(\boldsymbol{\xi}, \boldsymbol{\eta}) \widetilde{u}_{j}^{g}(\boldsymbol{\eta}) \mathrm{d} S(\boldsymbol{\eta})=\sum_{n=1}^{N_{f}^{g}} \int_{F_{n}^{g}} \widetilde{U}_{i j}^{g}(\boldsymbol{\xi}, \boldsymbol{\eta}) \widetilde{t}_{j}^{g}(\boldsymbol{\eta}) \mathrm{d} S(\boldsymbol{\eta}),
$$

225 with $i, j=1,2,3$, which can be written for each boundary nodal point belonging to the grain $g$. Eq. (5) expresses the displacement components $\widetilde{u}_{j}^{g}$ at the generic boundary collocation point $\boldsymbol{\xi} \in \mathscr{B}_{g}$ in terms of the displacements $\widetilde{u}_{j}^{g}(\boldsymbol{\eta})$ and tractions $\widetilde{t}_{j}^{g}(\boldsymbol{\eta})$ 
over the boundary $\mathscr{B}_{g}$ itself, with $\boldsymbol{\eta} \in \mathscr{B}_{g}$ being the generic boundary spanning integration point. The over-tilde $\sim$ denotes quantities expressed with respect to local reference systems defined over each grain's face $F_{n}^{g}$, thus varying as the integration point spans the grain's boundary, see e.g. Refs. [51, 54] and Fig. 33. $\widetilde{U}_{i j}^{g}(\boldsymbol{\xi}, \boldsymbol{\eta})$ and $\widetilde{T}_{i j}^{g}(\boldsymbol{\xi}, \boldsymbol{\eta})$ are combinations of the components of the 3D anisotropic fundamental sojutions of the grain material, computed as in Ref.[77] and briefly recalled in Appendix A $\widetilde{c}_{i j}^{g}(\boldsymbol{\xi})$ are free terms arising from the boundary limiting procedure and depend on the smoothness of the boundary $\mathscr{B}_{g}$ at the collocation point $\boldsymbol{\xi}$, see e.g. [76, 63].

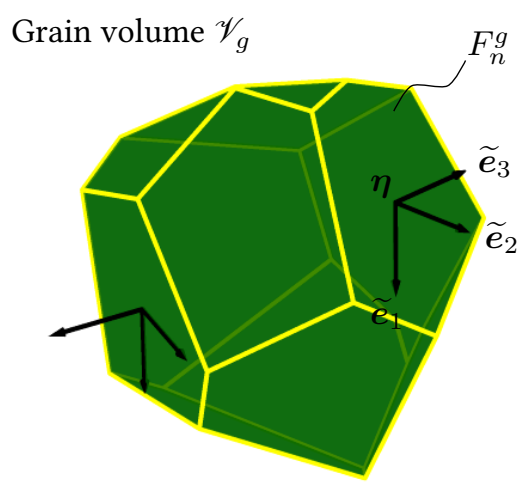

Figure 3: Each grain $g$ within the aggregate is a Voronoi polyhedron bounded by flat polygonal faces $F_{n}^{g}$; a local reference system $\left\{\widetilde{\boldsymbol{e}}_{1}, \widetilde{\boldsymbol{e}}_{2}, \widetilde{\boldsymbol{e}}_{3}\right\}$ is set over each face, to simplify the expression of the interface equations.

For the computational solution of the polycrystalline problem, Eq.55 is applied for each grain $g$ within the context of the boundary element method, according to the following scheme:

- Each grain face $F_{n}^{g}$ is subdivided into a collection of non-overlapping boundary elements, as briefly recalled in Section 2 and detailed in Ref.[55];

- The grain boundary displacement and traction fields are expressed through shape functions $\boldsymbol{N}_{e l}\left(\eta_{1}, \eta_{2}\right)$, defined over each element in a local 2D (surface) coordinate system $\left(\eta_{1}, \eta_{2}\right)$, and nodal values of boundary displacements $\widetilde{\boldsymbol{U}}^{g}$ and tractions $\widetilde{\boldsymbol{T}}^{g}$, expressed in the mentioned face-local reference systems; 
- Eq.(5) is collocated at each grain boundary node and it is numerically integrated, taking into account the approximation of the boundary fields in terms of shape functions and nodal values, as detailed in Ref.[55].

For each grain $g$ the above scheme leads to the system

$$
\widetilde{\boldsymbol{H}}^{g} \cdot \widetilde{\boldsymbol{U}}^{g}=\widetilde{\boldsymbol{G}}^{g} \cdot \widetilde{\boldsymbol{T}}^{g}
$$

expressed in terms of $3 \times N_{p}^{g}$ nodal values of displacements $\widetilde{\boldsymbol{U}}^{g}$ and $3 \times N_{p}^{g}$ nodal values of tractions $\widetilde{\boldsymbol{T}}^{g}$, if $N_{p}^{g}$ is the number of nodes associated with the grain $g$. The

${ }_{250}$ matrices $\widetilde{\boldsymbol{H}}^{g}$ and $\widetilde{\boldsymbol{G}}^{g}$ stem from the boundary integration of the kernels $\widetilde{T}_{i j}^{g}$ and $\widetilde{U}_{i j}^{g}$ respectively. Particular care must be used in the numerical integration of the elements containing the collocation point, as they give rise to singular integrals, see e.g. [76 63].

The application of the boundary conditions on the grain faces lying on the external walls of the aggregate transforms system $(6)$ into

$$
\boldsymbol{A}^{g} \cdot \boldsymbol{X}^{g}=\boldsymbol{C}^{g} \cdot \boldsymbol{Y}^{g}
$$

where the vectors $\boldsymbol{X}^{g}$ and $\boldsymbol{Y}^{g}$ collect respectively unknown and prescribed values of grain-boundary displacements and tractions, while the matrices $\boldsymbol{A}^{g}$ and $\boldsymbol{C}^{g}$ collect columns from $\widetilde{\boldsymbol{H}}^{g}$ and $\widetilde{\boldsymbol{G}}^{g}$ corresponding to the above unknown and prescribed degrees of freedom [63]. The terms $\boldsymbol{A}^{g}, \boldsymbol{X}^{g}, \boldsymbol{C}^{g}$ and $\boldsymbol{Y}^{g}$ are exactly those appearing in Eqs.(12).

\subsection{Intergranular interfaces modelling}

The matrix block $\boldsymbol{I}\left[\boldsymbol{X}, \boldsymbol{d}^{*}(N)\right]$ in Eq.11 implements the equations modelling the constitutive behaviour and progressive degradation of the intergranular interfaces. In this work, such interfaces are modelled using extrinsic cohesive traction-separation laws and the term $\boldsymbol{d}^{*}$ collects the components $d_{k}^{*}$ expressing the irreversible total damage accumulated at the $k$-th interface pair [54 55]. The use of cohesive laws for modelling fatigue degradation has been particularly explored in the composite materials community, see e.g. the review in Ref.[5], as their use is particularly suitable for tracking delamination in laminate configurations or lap joints. On the other hand, 
while cohesive laws have also been used for fatigue crack initiation in crystals [78, 79], other mechanisms related to slip accumulation within a grain are generally active in this class of materials [13]. In this work, attention is restricted to models for crystal aggregates in which the degradation processes are confined at the intergranular interfaces, see e.g. [47] and references therein.

In such cases, cyclic loads generally induce material degradation through nucleation, evolution and coalescence of micro-cracks and voids at the interfaces. In general, damage is accumulated at each loading cycle and several works have been devoted to the analysis of damage evolution on a cycle-by-cycle basis, resorting to loading-unloading hysteresis models [80, 81]. However, while such approaches are suitable for low-cycle applications, cycle-by-cycle analyses would be unfeasible in high-cycle applications, where the number of cycles to failure may overcome $N_{f}=$ $10^{5}$.

280 In high-cycle fatigue problems, the progressive degradation is usually expressed, in a macroscopic phenomenological framework, as a function of the number of loading cycles $N$ and of load and material parameters, e.g. load amplitude and/or range, fatigue activation threshold, etc. Broadly speaking, two different families of approaches can be identified in the literature: those based on continuum damage mechanics (CDM) 285 [9, 82] and those based on fracture mechanics (FM) [4]. CDM methods are based on the definition of a damage parameter, intended as a stiffness and/or strength reduction factor, whose evolution is directly expressed in terms of the number of cycles and loading and material parameters. On the other hand, FM approaches usually relate the crack growth rate $d a / d N$ to the range of fracture mechanics parameters, such as $\Delta K, \Delta G$ or $\Delta J$, through the well known Paris' law [10, 11] or its variants. These different approaches reflect sometimes a different focus, with CDM approaches addressed at the study of damage and crack initiation problems, in a safe-life design perspective, and FM approaches used for the analysis of crack propagation problems, within damage-tolerance design contexts.

295 More recently, a class of models based on cohesive zone modelling (CZM) has been developed for studying high-cycle fatigue in composite laminates [83, 84, 85, 86]. In these studies, cohesive traction-separation laws embodying irreversible damage pa- 
rameters are employed to model the effect of materials fatigue degradation. The damage variables evolve with the loading cycles according to specific evolution rules that may be stated either in a purely CDM framework [83 85] or in a purely FM framework [87], but also within the context of hybrid formulations based on the Paris' law, thus providing a link between CDM and FM [86 88].

\subsubsection{Intergranular damage decomposition}

In this study, we propose a CDM-based cohesive grain-boundary integral formulation for high-cycle intergranular fatigue in polycrystalline aggregates.

In CZM models for high-cycle fatigue, the total damage is usually decomposed into damage induced by quasi-static overloads and damage induced by cyclic loads [83, 86, 87, 88], so that the damage rate can be expressed as

$$
\frac{\partial d^{*}}{\partial N}=\frac{\partial d_{s}^{*}}{\partial N}+\frac{\partial d_{c}^{*}}{\partial N}
$$

where the differentiation is intended with respect to the number of load cycles $N$, considered as a continuous and differentiable time-like variable. Eq.8) can also be expressed in discrete terms as

$$
\Delta d^{*}=\Delta d_{s}^{*}+\Delta d_{c}^{*}
$$

where $\Delta d_{s}^{*}$ and $\Delta d_{c}^{*}$ are the quasi-static and cyclic contributions to the total damage $d^{*}$ over a cycle jump $\Delta N$. It is important to distinguish, here and in the subsequent sections, between the total damage and its quasi-static and cyclic contributions.

The decomposition given in Eq. (8) is assumed for the generic damage rate introduced in Eq. 3 and it will be used in conjunction with the envelope load approach and cycle jump strategy, both described in Section 3.4 for the solution of the high-cycle fatigue problem.

\subsubsection{Intergranular degradation under quasi-static loading}

The rules for the determination of the quasi-static contribution $\Delta d_{s}^{*}$ to the total damage are given in this section. The contribution $\Delta d_{s}^{*}$ after a cycles jump $\Delta N$ is determined once a static solution of Eq. 1 is is avalable. 
Let us consider the interface $\mathscr{I}^{a b}$ between two contiguous grains; a generic region $\mathscr{R} \in \mathscr{I}^{a b}$ may be pristine, damaged or failed/cracked. Let $P^{a}$ and $P^{b}$ denote a couple of homologous inter-granular points, belonging to the two contiguous grains $a$ and $b$ respectively. If the interface is locally in a pristine status, in the current loading conditions, then no relative displacements are allowed and the physical intergranular continuity requires

$$
\left\{\begin{aligned}
\delta \tilde{u}_{i}^{a b} & =-\left(\tilde{u}_{i}^{a}+\tilde{u}_{i}^{b}\right)=0 & & \text { displacements continuity } \\
\tilde{t}_{i}^{a} & =\tilde{t}_{i}^{b} & & \text { tractions equilibrium, }
\end{aligned}\right.
$$

for $i=1,2,3$.

Due to the external cyclic loading, each initially pristine interface experiences a local load cycle, in terms of intergranular tractions $\tilde{\boldsymbol{t}}^{a}=\tilde{\boldsymbol{t}}^{b}$. For such a reason, local intergranular damage nucleates and/or evolves at the interface, according to the CDM laws that will be discussed in Section 3.3.3 When intergranular damage $d^{*}$ arises at an interface, the continuity requirements expressed by Eqs.(10) are relaxed, and relative displacements between grain surfaces become possible. To model such a situation, Eqs. 10 are replaced by a cohesive model of the form

$$
\tilde{\boldsymbol{t}}=\boldsymbol{f}\left(\delta \tilde{\boldsymbol{u}}, d^{*}\right)
$$

where $\tilde{\boldsymbol{t}}^{T}=\left[\tilde{t}_{s}, \tilde{t}_{n}\right]$ collects the local tangential and normal traction components $\tilde{t}_{s}=\sqrt{\tilde{t}_{1}^{2}+\tilde{t}_{2}^{2}}$ and $\tilde{t}_{n}=\tilde{t}_{3}$, while $\delta \tilde{\boldsymbol{u}}^{T}=\left[\delta \tilde{u}_{s}, \delta \tilde{u}_{n}\right]$ with $\delta \tilde{u}_{s}=\sqrt{\delta \tilde{u}_{1}^{2}+\delta \tilde{u}_{2}^{2}}$ and $\delta \tilde{u}_{n}=\delta \tilde{u}_{3}$. The local total damage $d^{*} \in[0,1]$ accounts for the amount of local intergranular degradation. In this study, the cohesive law in Eq.11 is assumed of the form

$$
\tilde{\boldsymbol{t}}=\boldsymbol{K}\left(d^{*}\right) \delta \tilde{\boldsymbol{u}},
$$

with

$$
\boldsymbol{K}\left(d^{*}\right)=T_{0} \frac{1-d^{*}}{d^{*}}\left[\begin{array}{cc}
\frac{\alpha}{\delta u_{s}^{c r}} & 0 \\
0 & \frac{1}{\delta u_{n}^{c r}}
\end{array}\right],
$$

where $T_{0}$ is the local intergranular cohesive strength, $\delta u_{n}^{c r}$ and $\delta u_{s}^{c r}$ are the local critical displacement openings in pure mode I and pure mode II, respectively, and $\alpha$ is a constant weighting mode I and mode II [53 54]. Eqs.12 13] have been used in Refs. 
[53 54 55] to study progressive damage and failure evolving under the action of quasistatic loads. They express a cohesive law isotropic with respect to the directions of sliding over the contact surface and with uncoupled opening and sliding modes. They allow to model mixed-mode loading and can capture local loading, unloading and reloading: during loading, the quasi-static damage at the interface increases under external driving forces; in unloading and reloading the damage is constant and the interface behaves elastically, with the tractions decreasing in case of unloading or increasing in the case of reloading, up to the maximum allowed value before re-entering a loading stage.

It important to realise that the damage $d^{*}$ appearing in Eqs. 11 13) is the total damage, while the method for determining the quasi-static contribution is given next. At a generic solution step, identified by the number of cycles $N$, the total damage $d^{*}(N)$ is accumulated at the generic intergranular region. As the number of load cycles increases from $N$ to $N+\Delta N$, the damage evolves and spreads within the polycrystalline aggregate and the overall microstructure becomes more compliant. As a consequence, when Eq.(1) is solved at $N+\Delta N$, due to the redistribution of internal stresses and intergranular tractions, further damage evolution can be promoted by the quasi-static overloads associated to the external loads.

In this study it is assumed that local quasi-static damage evolution is triggered when the effective opening displacement $d$, defined by

$$
d=\left[\left\langle\frac{\delta \tilde{u}_{n}}{\delta u_{n}^{c r}}\right\rangle^{2}+\beta^{2}\left(\frac{\delta \tilde{u}_{s}}{\delta u_{s}^{c r}}\right)^{2}\right]^{\frac{1}{2}},
$$

overcomes the local quasi-static damage $d_{s}^{*}(N)$, accumulated so far at the considered interfacial region. At each solution step, the computation of $d$ is possible as both $\delta \tilde{u}_{s}$ and $\delta \tilde{u}_{s}$ are available as direct solution of the polycrystalline system given in Eq.(1), while $\beta$ is a cohesive constant weighting mode I and mode II. The interfaces where $d>d_{s}^{*}$ experience thus local loading, with further increase of the quasi-static damage contribution $d_{s}^{*}$ obtainable from

$$
d_{s}^{*}(N+\Delta N)=\max \left\{d(N+\Delta N), d_{s}^{*}(N)\right\} \in[0,1] .
$$

It is worth noting that the need to assess Eq.(15) requires the storage of the current 
value of static damage for each interface pair.

In the above scenario, an already damaged interface, modelled by Eqs. 11. 13, may develop additional damage under the action of quasi-static overloads. However, a different potential situation may arise at some interfaces, especially during the initial cycles of relatively high loads, i.e. loads able to induce intergranular tractions close to the cohesive interfacial strength $T_{0}$. It may happen that a pristine interface, modelled by the continuity conditions Eqs. 10 , experiences a level of tractions high enough to directly initiate quasi-static damage. This situation is met when the condition

$$
t_{e}=\left[\left\langle\tilde{t}_{n}\right\rangle^{2}+\left(\frac{\beta}{\alpha} \tilde{t}_{s}\right)^{2}\right]^{\frac{1}{2}} \geq T_{0}
$$

holds at a pristine interface, where $t_{e}$ is an effective traction, $\tilde{t}_{s}=\sqrt{\tilde{t}_{1}^{2}+\tilde{t}_{2}^{2}}$ is the tangential traction component, $\tilde{t}_{n}=\tilde{t}_{3}$ is the normal traction component and $\langle\cdot\rangle$ denote the Macauley's brackets. If Eq. 16 is met at an interface, Eqs.10 are dropped and the extrinsic cohesive laws given in Eqs.12 13) are introduced, with an initially small guess value of intergranular damage $d_{s}^{*}$ then subjected to iteration, as further specified in Section 3.4 .

The local intergranular value of total damage $d^{*}$ increases monotonically between 0 and 1 . When the critical condition $d^{*}=1$ is reached, intergranular failure is reached and the cohesive laws no longer apply. At this point, frictional contact is introduced to model the micro-cracks, whose surfaces may separate, slip or stick. Upon failure, the cohesive-frictional laws proposed in Ref.[54] are employed.

\subsubsection{Intergranular degradation under cyclic loading}

The rules for updating the cyclic components $d_{c}^{*}$ of damage are discussed in this section. In this work, the intergranular degradation is framed in a continuum damage mechanics background [89, 90, 91, 9, 92, 93]. In particular, according to Xiao et al. [92], and especially to Jalalahmadi et al. [28] and Bomidi et al. [47], who also studied fatigue degradation in polycrystalline microstructures with confined plasticity, the law employed to express the fatigue damage rate, at a generic interface pair, is assumed 
as

$$
\frac{\partial d_{c}^{*}}{\partial N}=\left[\frac{\llbracket \hat{t} \rrbracket}{\sigma_{r}\left(1-d^{*}\right)}\right]^{m},
$$

where $d^{*}$ is the current total locally accumulated damage, $\sigma_{r}$ is a material parameter referred to as resistance stress, $m$ is a material parameter to be experimentally identified and $\llbracket \hat{t} \rrbracket$ represents the range of a cyclic intergranular traction responsible for fatigue degradation, i.e. an effective intergranular traction range, which should be suitably identified. Bomidi et al. [47] suggest that it should be determined according to the considered loading conditions and in their work they selected the components of stress normal to the local intergranular interfaces, which here would correspond to $\llbracket \tilde{t}_{n} \rrbracket$. In the present framework, it is reasonable to assume, in principle, a more general dependence, taking also into account the influence of the tangental tractions on the fatigue degradation. The effective range may be then defined as

$$
\llbracket \hat{t} \rrbracket=\sqrt{\llbracket \tilde{t}_{n} \rrbracket^{2}+\gamma \cdot \llbracket \tilde{t}_{s} \rrbracket^{2}}
$$

where $\gamma$ is an effective range weight parameter to be suitably identified for the considered material and load conditions.

Since the amplitude of a generic traction $\llbracket t \rrbracket=t_{\max }-t_{\min }=t_{\max }(1-R)$, where $t_{\max }$ and $t_{\min }$ are the maximum and minimum values over a load cycle and $R=t_{\min } / t_{\max }$, Eq.17 can be expressed for the overall aggregate, collecting the components of cyclic damage for all the interface pairs, in the general functional form

$$
\frac{\partial \boldsymbol{d}_{c}^{*}}{\partial N}=\Psi_{c}\left(\boldsymbol{X}, \boldsymbol{R}, \boldsymbol{d}^{*}, \boldsymbol{\pi}\right),
$$

355 where it has been considered that the tractions are a subset of the unknown vector $\boldsymbol{X}$ in Eq.11 and $\boldsymbol{R}$ is representative of the load ranges over the individual interfaces. In other words, since the amplitude can be expressed in terms of the maximum of a variable over a load cycle and, as it will be discussed next, in an envelope load representation the maxima of the intergranular tractions are a direct output of a solution step, Eq. 19 can be directly expressed in terms of the solution $\boldsymbol{X}$ of the boundary value problem. Eq. 19 will be used in the discussion that follows, where it will be explained how such evolution rule plays the role of updating damage in a cycle jump. 


\subsection{Fatigue solution algorithm}

The overall procedure for the analysis of polycrystalline aggregates undergoing high-cycle fatigue intergranular degradation is described here.

Since in high-cycle fatigue problems the explicit simulation of individual cycles is computationally too expensive or unfeasible, the damage decomposition in Eq. 8 is often employed in conjunction with an envelope load representation [82, $83,85,87,88]$ and a cycle jump strategy [94, 86, 28, 87].

The envelope load representation consists in loading the system considering only the envelope of the load cycles maxima, see e.g. Refs. [84 5], based on the consideration that most cyclic damage is induced during the loading phase of each cycle [82].

On the other hand, in the cycle jump strategy the system is assessed at discrete 375 cycles intervals $\left\{N_{1}, N_{2}, \ldots, N_{k}, \ldots\right\}$, with $N_{k+1}=N_{k}+\Delta N_{k}$, and it is assumed that between $N_{k}$ and $N_{k+1}$ the system is subjected to the cyclic action of $\Delta N_{k}$ cycles. If the problem solution at $N_{k}$ is known, the system is sampled at $N_{k+1}$, after $\Delta N_{k}$ cycles during which it has experienced cyclic degradation. At $N_{k+1}$ it will then be more compliant and the quasi-static damage evolution rules are assessed.

In other words, the specimen is alternately loaded by quasi-static and cyclic steps: in a quasi-static step, at a generic $N_{k}$, the structure is subjected to the maximum value of load corresponding to $N_{k}$ (envelope load, either force or displacement), and the damage $d_{s}^{*}$ is evolved according to the quasi-static cohesive damage rules, upon numerical solution of the underlying structural finite/boundary element problem; in a cyclic step, between $N_{k}$ and $N_{k+1}$, cyclic damage $d_{c}^{*}$ is evolved according to rate laws formulated on the basis of different principles [82, 83, 86, 28, 47, 88]. It is worth noting that, during a cyclic step, the fatigue degradation evolved over $\Delta N_{k}$ cycles is estimated employing evolution laws depending on the total damage accumulated so far, so that the accumulation of damage is inherently coupled. A schematic diagram of the adopted solution strategy is shown in Fig.44.

It has been observed that, in high-cycle envelope-load cycle-jump strategies, the quasi-static step allows the redistribution of stresses/strains, following the progressive material degradation, by fulfilling the equilibrium equations through the system 

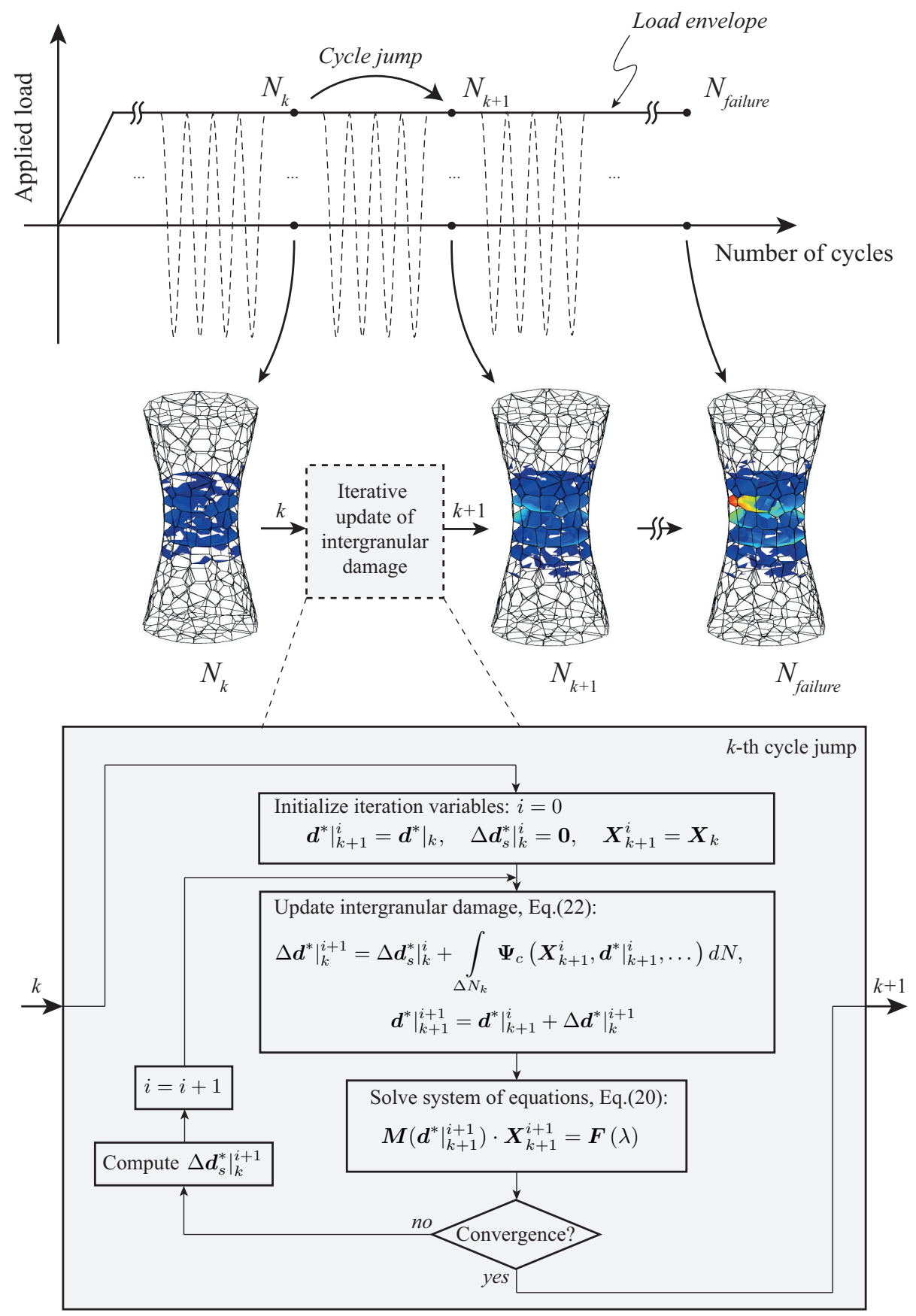

Figure 4: Schematic diagram of the adopted solution strategy. 
solution, while the cycles jump plays the role of a simplified one-point Newton-Cotes quadrature: in this strategy, an accuracy control can be introduced by limiting the amount of damage $\Delta d_{c}^{*}$ that can be accumulated at each interface pair $k$ during a generic cycles jump, which in turn limits the number of cycles $\Delta N$ that can be applied during a cyclic loading step [94 86]. On the other hand, more refined iterative quadrature schemes [83, 85] can be used to enhance the accuracy of the extrapolated 400 fatigue damage in a cycle jump. In this work, for the integration of the polycrystalline fatigue problem, we employ a strategy similar to that used by Robinson et al. 83]. An in-depth critical review on the features of the different formulations used for fatigue delamination in composite laminates may be found Ref.[5].

The details about the numerical integration of the problem are now discussed. Considering the form of the cohesive laws in Eq.122, the system in Eq.11 can be rewritten

$$
\boldsymbol{M}\left[\boldsymbol{d}^{*}(N)\right] \cdot \boldsymbol{X}=\boldsymbol{F}(\lambda) .
$$

The previous equation must be coupled with the evolution law in Eq.8 that, considering Eq.19], can be rewritten

$$
\frac{\partial \boldsymbol{d}^{*}}{\partial N}=\frac{\partial \boldsymbol{d}_{s}^{*}}{\partial N}+\Psi_{c}\left(\boldsymbol{X}, \boldsymbol{R}, \boldsymbol{d}^{*}, \boldsymbol{\pi}\right) .
$$

Since an explicit expression for the quasi-static damage rate is not available, it is convenient to integrate the previous equation over a generic cycle jump $N_{k} \rightarrow N_{k+1}=$ $N_{k}+\Delta N_{k}$, following a procedure similar to that discussed in Ref.[83], obtaining

$$
\Delta \boldsymbol{d}^{*}=\Delta \boldsymbol{d}_{s}^{*}+\int_{\Delta N_{k}} \boldsymbol{\Psi}_{c}\left(\boldsymbol{X}, \boldsymbol{R}, \boldsymbol{d}^{*}, \boldsymbol{\pi}\right) d N,
$$

where $\Delta \boldsymbol{d}_{s}^{*}$ can be computed using the rules given in Section 3.3 .2 and $\boldsymbol{\Psi}_{c}$ can be integrated using the explicit expression in Eq.(17). It is worth noting that, since $\boldsymbol{X}$ and $\boldsymbol{d}^{*}$ explicitly enter the rate laws, the coupled Eq.20 and Eq.(22) must be solved iteratively over a cycle jump. In this work, an implicit backward Euler scheme is used in the incremental/iterative solution procedure.

Since the matrix $\boldsymbol{M}\left[\boldsymbol{d}^{*}(N)\right]$ is highly sparse, Eq. 20$]$ is solved using PARDISO (http : / /www . pardiso-project . org/) [95 96, 97]. In the solution of Eq. 20, 
which stems from a boundary element collocation procedure, higher computational efficiency could be attained using Krylov iterative solvers in conjunction with special matrix representations, obtained for example using fast multipoles [98] or hierarchical matrices [99 100, 101].

\section{$415 \quad$ 4. Numerical tests}

In this section, the developed formulation is applied to the study of intergranular degradation of pseudo and fully 3D polycrystalline components under cyclic loading. All tests have been performed on CINECA's Marconi's HPC infrastructure, on single nodes with 36 cores and 128 GB RAM.

\subsection{Pseudo-3D components}

Pseudo-3D polycrystalline specimens with external shape as shown in Fig.(5) are first considered. The external geometry is completely identified by $a=28.0 \mu \mathrm{m}$, $b=a / 2=14.0 \mu \mathrm{m}, c=3 a / 10=8.4 \mu \mathrm{m}$ and $d=a / 10=2.8 \mu \mathrm{m}$, the thickness $t h=0.7 \mu \mathrm{m}$ and by considering that the curved walls have parabolic curvature.

The grains are generated extruding a 2D tessellation in the thickness direction, from which the adjective pseudo- $3 D$, but no restriction is enforced on the crystallographic orientation of the individual grains. Five different tessellations have been generated using the procedure described in Section 2 starting from an initial prismatic $a \times b \times t h$ box containing $N_{i}=500$ original seeds. After seeds selection, mirroring and grains removal, the different specimens contained a number of grains $336 \leq N_{g} \leq 368$, corresponding to an average grain size $\bar{d}_{g}=1 \mu \mathrm{m}$ [102].

Polysilicon is considered as material: the bulk grains present cubic material symmetry with elastic constants $c_{11}=166 \mathrm{GPa}, c_{12}=64 \mathrm{GPa}, c_{44}=79.6 \mathrm{GPa}$, according to Ref.[102]. The intergranular interfaces are fully characterised by the properties summarised in Table 1 which have been estimated as follows. The value of the intergranular cohesive strength $T_{0}$ is closely related to the bulk material macroscopic strength, as shown in previous works [54 55 57] and the value $T_{0}=550$ MPa has been selected by considering the static strength of polysilicon as reported in 
Refs. [102 47]. The value of the critical displacement jump $\delta u_{n}^{c r}$ for mode I has been estimated by considering the relationship between the work of separation $G_{I}$, the fracture toughness $K_{I}$ and the elastic constants $E$ and $\nu$ of the bulk material [54], which reads

$$
G_{I}=\frac{1}{2} T_{0} \delta u_{n}^{c r}=\frac{\left(1-\nu^{2}\right) K_{I}^{2}}{E} .
$$

In this work, $E=163 \mathrm{GPa}$ and $\nu=0.22$ are the elastic constants of bulk polysilicon, estimated by computing the Hashin-Shtrikman bounds [103] of a random polycrystalline aggregate of grains with the single crystals properties $c_{11}, c_{12}$ and $c_{44}$ as given above; $K_{I}=0.94 \mathrm{MPa} \sqrt{\mathrm{m}}$ is the fracture toughness for polysilicon consistent with values reported in Ref.[31]. The values of the shear critical displacement $\delta u_{s}^{c r}$ and of the constants $\alpha$ and $\beta$ are selected considering $G_{I}=G_{I I}$, as done in Ref.[54]. Eventually, $\sigma_{r}$ and $m$ are taken from Ref.[47], while $\gamma$ is selected so as to give the same relative weight between normal and sliding tractions in the definition of the effective range, Eq.18), as that assigned in the definition of the static effective traction, Eq.(16).

The specimens are subjected to the boundary conditions represented in Fig.(5): they are clamped on the left-hand side, $\boldsymbol{u}=\mathbf{0}$, and can be subjected to generic displacements $\boldsymbol{u}=\overline{\boldsymbol{u}}$ or tractions $\boldsymbol{t}=\overline{\boldsymbol{\sigma}}$ on the right-hand side. It is worth recalling that, being the envelope load strategy employed in this formulation, only the maxi-

Table 1: Intergranular properties for the simulated polycrystalline components.

\begin{tabular}{lcl}
\hline \hline Property & Component & Value \\
\hline Interface strength & $T_{0}$ & $550 \mathrm{MPa}$ \\
Cohesive law constants & $\alpha$ & 1 \\
& $\beta$ & $\sqrt{2}$ \\
Critical displacements jumps & $\delta u_{n}^{c r}$ & $1.89 \cdot 10^{-2} \mu \mathrm{m}$ \\
Cyclic damage rate resistance stress & $\delta u_{s}^{c r}$ & $\left(\beta^{2} / \alpha\right) \delta u_{n}^{c r}$ \\
Cyclic damage rate exponent & $\sigma_{r}$ & $2668 \mathrm{MPa}$ \\
Effective range weight parameter, see Eq. 18 & $m$ & 8.93 \\
\hline \hline
\end{tabular}




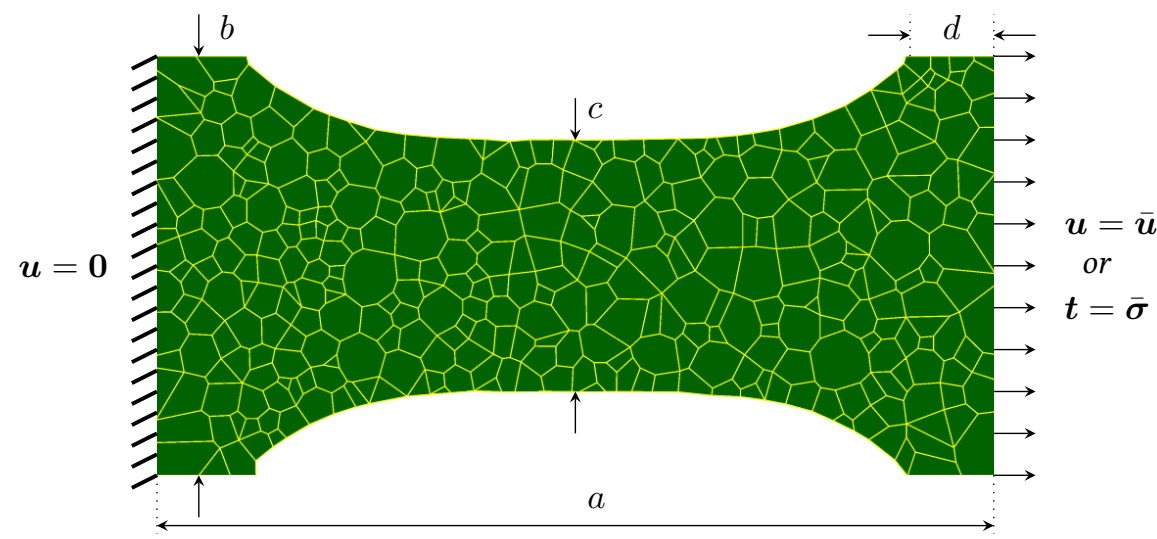

Figure 5: Geometry and boundary conditions for the simulated pseudo-3D polycrystalline components. Five different polycrystalline tessellations of the shown geometry are considered. The specimens are clamped on the left side and are subjected to either displacement or traction boundary conditions on the right side. The remaining external surfaces are traction-free.

mum value of the enforced field, either displacement or traction, is taken into account in the identification of the boundary conditions and no load cycle is ever explicitly simulated. In other words, if the generic boundary condition $f=\bar{f}$ is enforced in the boundary value problem, this corresponds to enforcing a cyclic load with amplitude $\llbracket f \rrbracket=(1-R) \bar{f}$, with $R$ suitably selected for the considered cyclic load, e.g. $R=-1$ for a fully reversed load.

In order to calibrate the high-cycle tests, in terms of applied cyclic load, the polycrystalline components are first subjected to progressive quasi-static loading: Fig.6] shows the average applied stress versus nominal strains for quasi-static loading in displacement control. The obtained results show that all the morphologies exhibit very similar stress-strain behaviour as well as failure strain and stress: in this sense it is meaningful to refer to the component, almost irrespective of the underlying polycrystalline morphology, at least for the considered number of grains. It is worth highlighting that here the focus is not on the statistical computational homogenisation of polycrystalline aggregates. An in-depth investigation about the determination of ${ }_{460}$ representative volume elements for polycrystalline materials, using the baseline integral formulation employed here, has been performed in Refs. [54, 55 [56], where it 


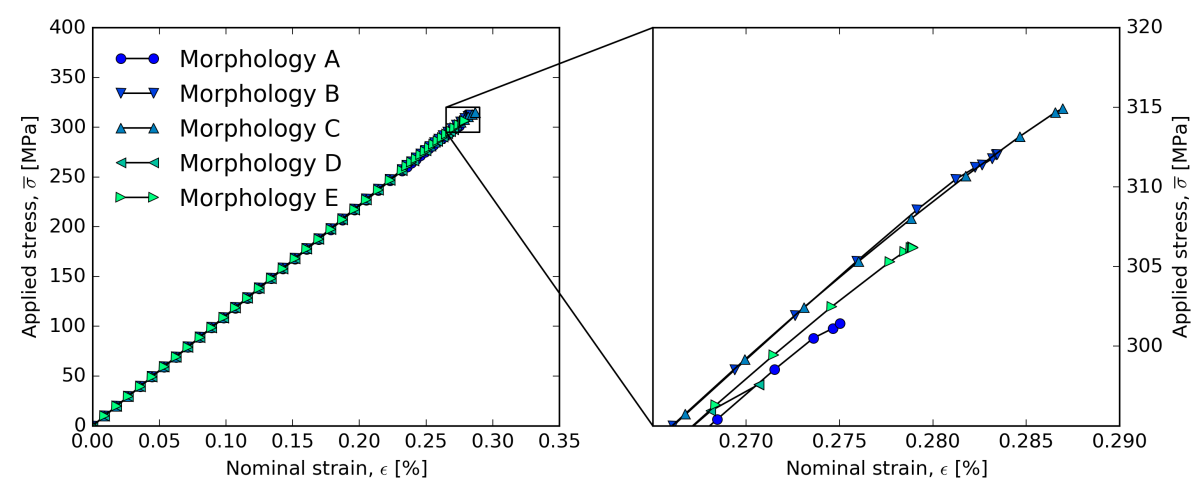

Figure 6: Average applied stress versus nominal strain for the five morphologies under progressive quasistatic loading in displacement control: all the morphologies exhibit similar stress-strain behaviour and strain and stress at failure.

was shown that few hundred grains are sufficient to attain representative aggregates for materials with levels of single-crystal anisotropy as those considered in this study, which is the reason why the stress-strain curves appear close to each other. The curves shown in Fig.66 are then employed to set the boundaries for the high-cycle tests in terms of applied cyclic stress: referring to Fig.(5), the applied cyclic amplitude can be expressed as $\llbracket t \rrbracket=(1-R) \bar{\sigma}$, with $\bar{\sigma}<\sigma_{s f}$, where $\sigma_{s f}$ is the quasi-static failure stress.

Fig.(7a) shows the average damage versus the total number of cycles for the five different morphologies subjected to fully reversed cyclic stress $\bar{\sigma}$. Fig.(7p), on the other hand, shows the relative weight of the accumulated quasi-static and cyclic contributions upon failure for morphology A. It is noted that, as $\bar{\sigma}$ decreases, resulting in higher cycle fatigue conditions, the cumulative cyclic damage contribution becomes predominant upon failure, while the quasi-static contribution is predominant when $\bar{\sigma}$ is closer to the static failure strength, as it appears physically consistent.

The average total damage is defined as

$$
D \equiv \frac{1}{S_{I}} \int_{S_{I}} d^{*}(\boldsymbol{x}) \mathrm{d} S(\boldsymbol{x}),
$$

where the integral of the local damage $d^{*}(\boldsymbol{x})$ is computed over all the intergranular interfaces $S_{I}$. It is shown how the amplitude of the applied load affects the fatigue life 
Table 2: Computed maximum and minimum numbers of cycles for the analysed morphologies at different load levels.

\begin{tabular}{ccccc}
\hline \hline $\bar{\sigma}$ & $250 \mathrm{MPa}$ & $200 \mathrm{MPa}$ & $150 \mathrm{MPa}$ & $100 \mathrm{MPa}$ \\
\hline$N_{\min }$ & 130 & 4108 & 103581 & 5816070 \\
$N_{\max }$ & 337 & 6232 & 142440 & 7585490 \\
$\Delta N$ & 207 & 2124 & 38859 & 1769420 \\
\hline \hline
\end{tabular}

of the component: the components subjected to lower amplitudes survive considerably longer than those loaded with higher values. It is also shown how, in all the tests, the components failure is reached for values of average total damage that may appear relatively low: however, this is due to the averaging process, that is also extended over interfaces that experience little or no damage, as those located in the sections closer to the larger lateral sides. However, as shown in Fig.80, which shows the damage patterns for four of the analysed morphologies under the action of $\bar{\sigma}=100 \mathrm{MPa}$, it is actually true that failure is triggered when a relatively low level of local damage is reached in critical locations: this is a consequence of the brittleness of the analysed specimens and of the specific loading conditions, namely load control, which trigger failure as soon as the residual fracture energy of the damaged interfaces is overcome by the stored strain energy during a quasi-static load step. Such considerations agree with those reported in Ref.[47], who observed that damage initiation and failure actually coincided in their study, in which the same material as that considered here was analysed. Fig.(8), on the other hand, shows the crack configuration at the last computed cycle jump for four of the analysed tessellations, which are anyway representative of the behaviour found in all the specimens.

${ }_{495}$ Eventually, Fig. 9 shows the $\bar{\sigma}-N$ curve for the analysed components: as expected, as the amplitude of the applied load increases, the number of cycles to failure decreases. Table 2 reports the maximum and minimum numbers of cycles for the analysed morphologies at different load levels: it can be noted as the scatter of fatigue life increase when the level of applied load decreases, i.e. when conditions of high-cycle fatigue are met. 


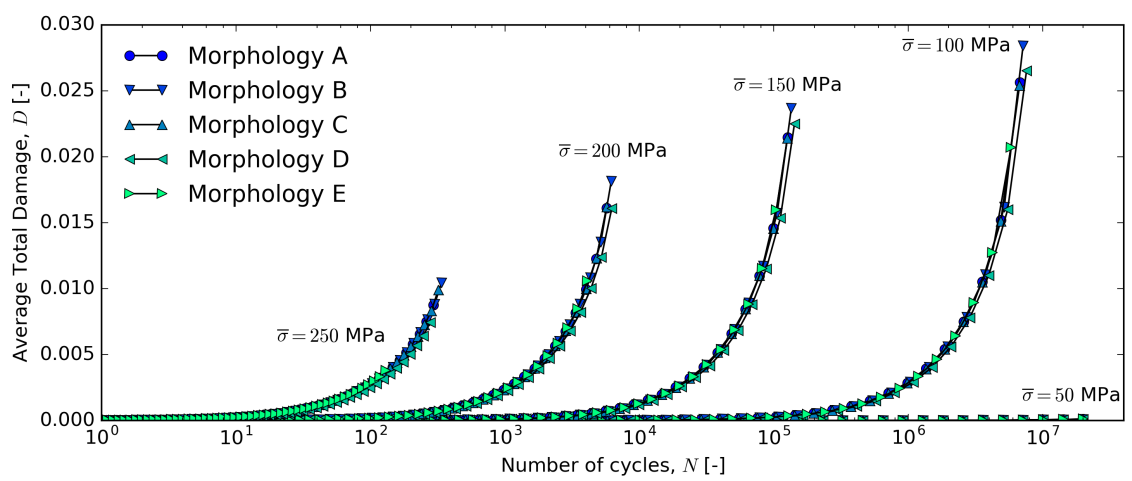

(a)

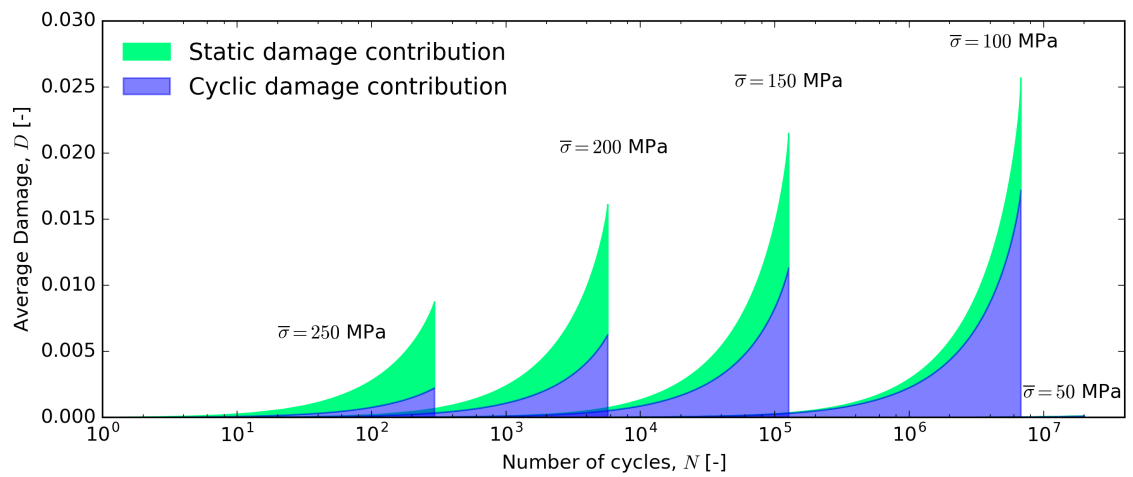

(b)

Figure 7: Plots of average damage versus number of cycles for the considered morphologies subjected to fully reversed cyclic load at different levels of load amplitude: a) Average total damage versus number of cycles for the five different morphologies; b) Cyclic and quasi-static average damage contributions for morphology A.

\subsection{Fully 3D components}

Five different fully 3D non-convex morphologies are considered in this section. The morphologies have been generated within the non-convex bounding surface $\mathscr{B}$, obtained by revolution of the parabolic profile $r(z)$ around the $z$ axis, as shown in Fig. 10a). One of the resulting tessellations is shown in Fig.10b; the tessellations contain a number of grains $436 \leq N_{g} \leq 482$, corresponding to an average grain size $\bar{d}_{g}=1 \mu \mathrm{m}$ [102]. The specimens are clamped at the bottom end and are loaded by spatially uniform tractions directed along $z$ and acting on the top end. 

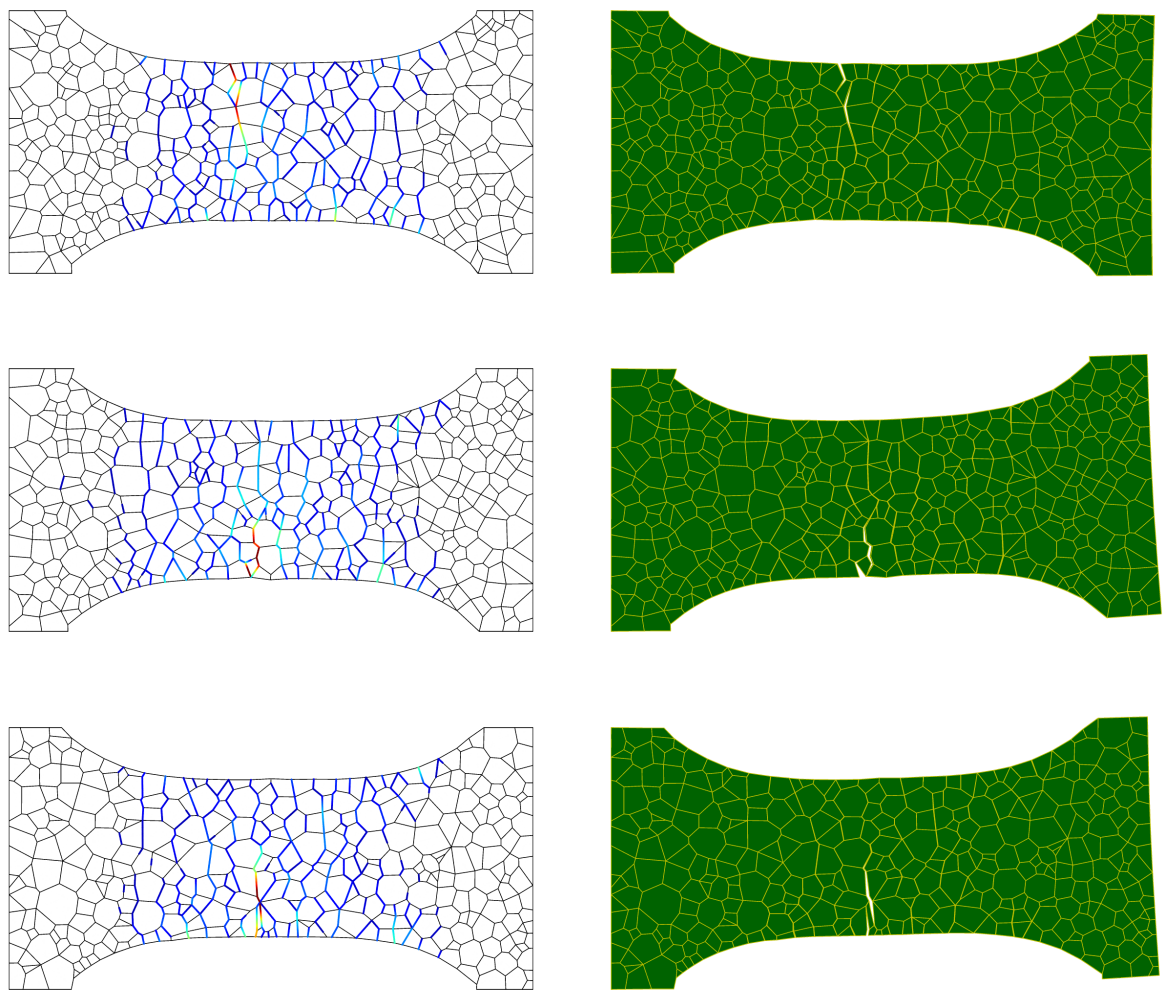

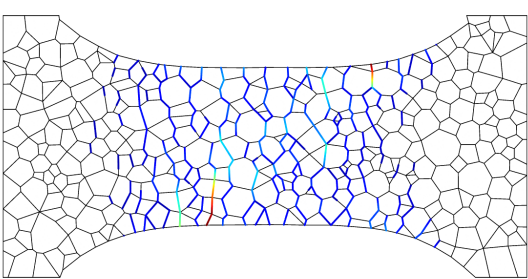

(a)

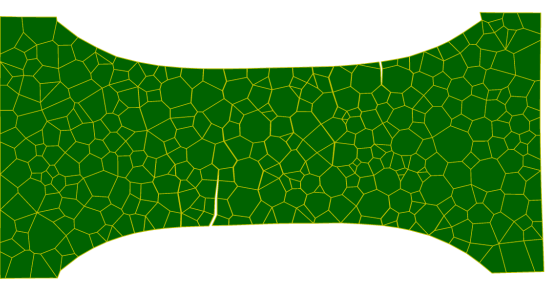

(b)

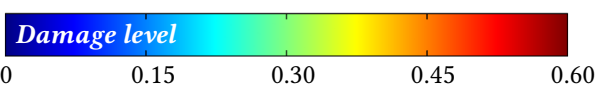

Figure 8: Damage ( $a$ ) and crack $(b)$ patterns at the last computed cycle jump before failure for the pseudo3D morphologies A-D under the action of $\bar{\sigma}=100 \mathrm{MPa}$ (the amplitude for fully reversed cycles is then $200 \mathrm{MPa}$ ); the shown behaviour is representative of that of all the morphologies. 


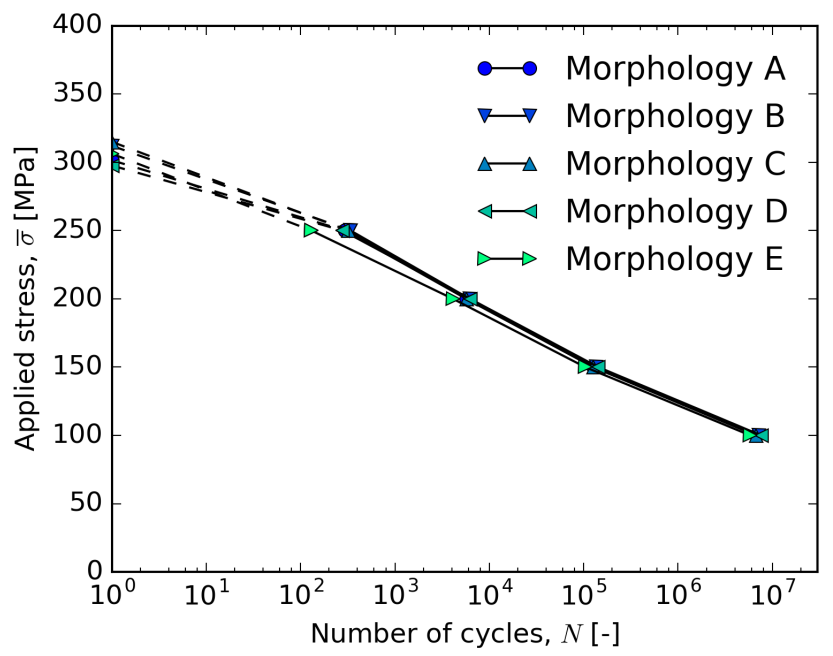

Figure 9: $\bar{\sigma}-N$ curves for the analysed pseudo-3D polycrystalline components.

Before simulating the cyclic loading, the 3D specimens are analysed under the action of progressive quasi-static loads, analogously to what has been done for the pseudo-3D case, in order to identify appropriate values for the range of the applied cyclic tractions $\bar{\sigma}$.

Fig.111) reports the plot of the average damage, as defined in Eq.24, versus the number of load cycles for the analysed morphologies. Five different morphologies are first analysed under the action of a cyclic load $\bar{\sigma}=100 \mathrm{MPa}$ (then amplitude $200 \mathrm{MPa}$ ) and it is found that the five aggregates show comparable behaviour in terms of total damage versus number of cycles. However, it is worth noting that, as already observed in the pseudo-3D case, a certain level of fatigue life scatter is present: in this case, for the considered morphologies at $\bar{\sigma}=100 \mathrm{MPa}, N_{\min }=74671$ and $N_{\max }=444901$. ${ }_{520}$ A thorough investigation and statistical characterisation of the fatigue life scatter is beyond the scope of the present paper: however, interested readers are referred to Refs. [28 47], where a Weibull probability analysis of fatigue life of polycrystalline specimen is presented. The aggregate $\mathrm{A}$ is then tested at different values of $\bar{\sigma}$ and similar conclusions as those for the pseudo-3D tests can be drawn. Fig.(11p), on the other hand, reports the relative weight of quasi-static and cyclic damage contributions 


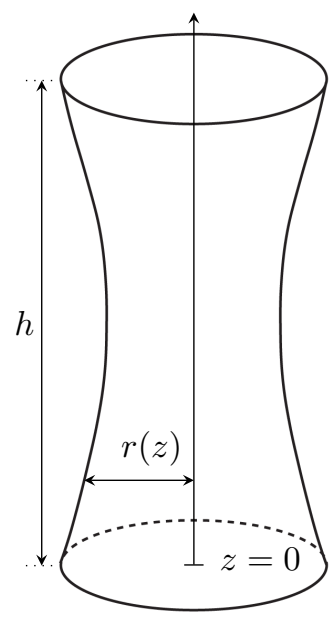

(a)

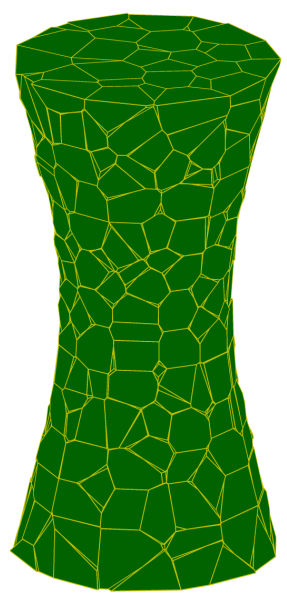

(b)

Figure 10: Geometry $(a)$ and tessellation $(b)$ for the simulated fully 3D polycrystalline components. Five different polycrystalline tessellations of the shown geometry are considered. The specimens are clamped at the bottom $(z=0)$ end and are subjected to cyclic uniform fully reversed $(R=-1)$ tractions directed along $z$ and acting over top end $(z=h=10.6 \mu \mathrm{m})$. The remaining external surfaces are traction-free.

and similar conclusions as those for the pseudo-3D case can be drawn.

Fig.(12) shows the damage and crack patterns at the last computed cycle jump for three of the analysed morphologies under the action of $\bar{\sigma}=100 \mathrm{MPa}$. Similar conclusions as those drawn for the previously considered pseudo-3D tests hold.

Eventually, Fig.(13) shows the $\bar{\sigma}-N$ curve for the morphology A, which appears consistent with physical expectations.

\section{Discussion}

A microstructural model for high-cycle fatigue degradation in polycrystalline aggregates has been proposed in this work. The distinguishing feature of the framework is that it is based on a boundary integral representation of the mechanical problem for the individual crystals, which allow to formulate the overall problem in terms of intergranular variables, i.e. displacement jumps and tractions, thus allowing $a$ ) a particularly effective expression of the intergranular cohesive traction-separation laws, 


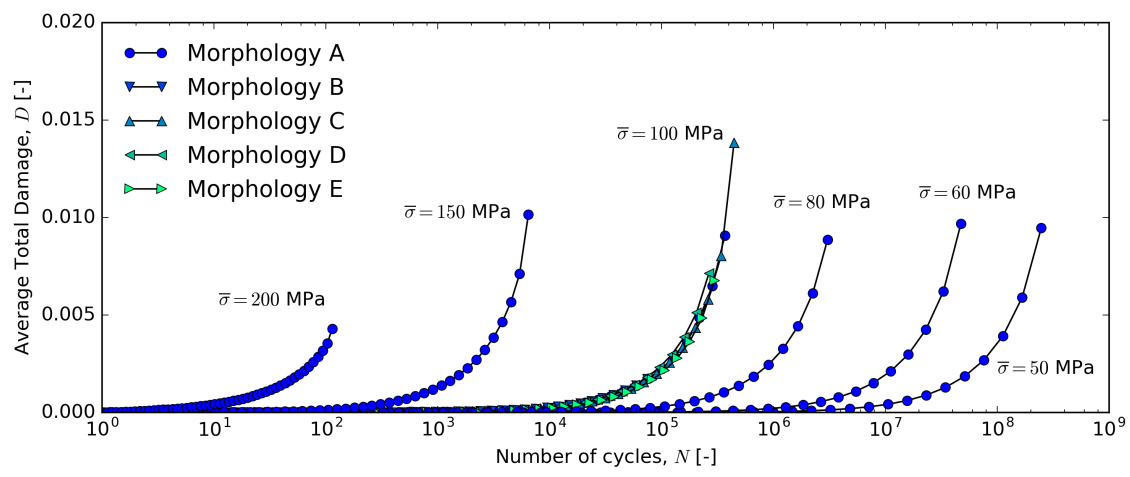

(a)

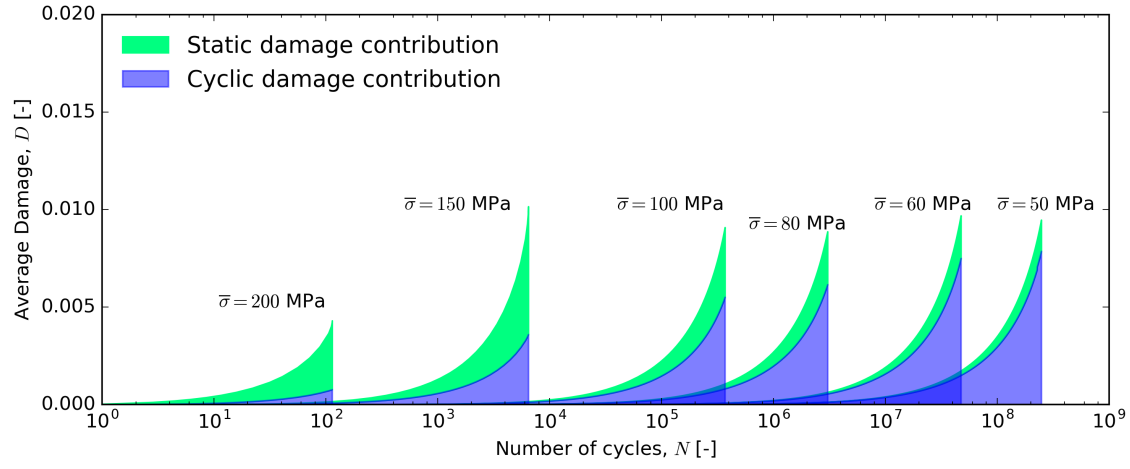

(b)

Figure 11: Plots of average damage versus number of cycles for the considered morphologies subjected to fully reversed cyclic load: a) Average total damage versus number of cycles: all the five morphologies have been tested for a load amplitude $\bar{\sigma}=100 \mathrm{MPa}$, which shows a good level of reproducibility; morphology A has then been tested at different levels of load amplitude, as shown; b) Cyclic and quasi-static average damage contributions for morphology $\mathrm{A}$ at different levels of load amplitude; the cyclic contribution weight increases as the level of load amplitude decreases, as seen also in the pseudo-3D case.

b) a relative reduction in the number of DoFs with respect to volume discretisation methods (also when crystal plasticity is considered for the grains, [52]) and c) a relative simplification in input data preparation.

Several directions of further development can be identified for the proposed framework, from the formulation of specific cohesive laws for the analysis of low-cycle fatigue problems [80, 81] to the inclusion of slip-accumulation mechanisms within the 


$\begin{array}{lrrrr}0 & 0.15 & 0.30 & 0.45 & 0.60 \\ \text { Damage level } & & ,\end{array}$
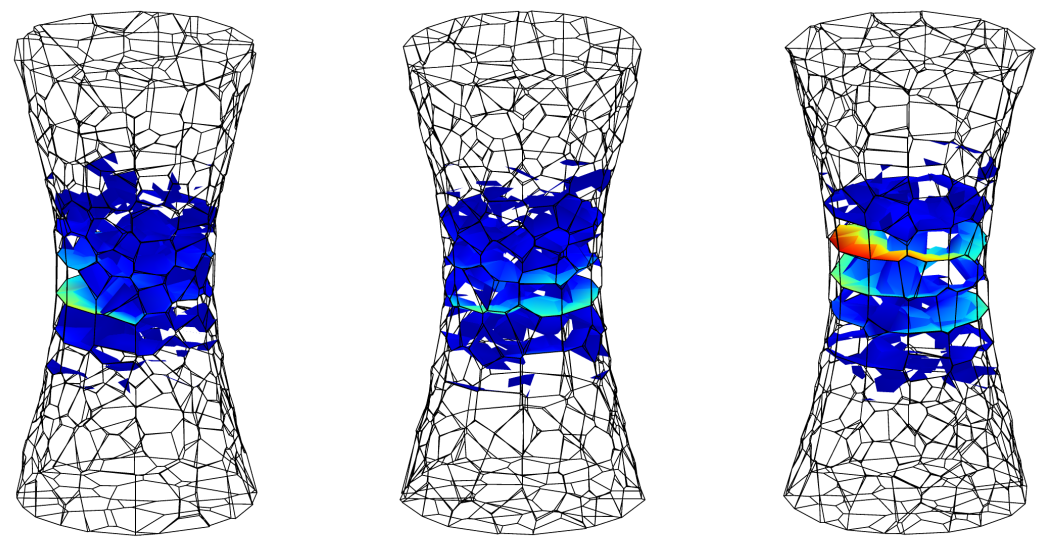

(a)
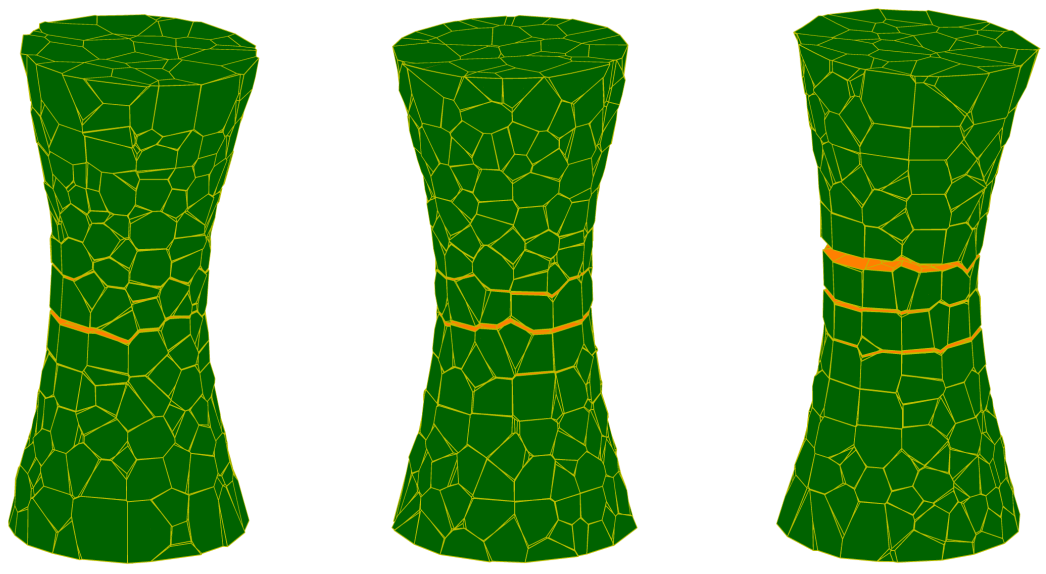

(b)

Figure 12: Damage ( $a$ ) and crack $(b)$ patterns at the last computed cycle jump before failure for the fully 3D morphologies A, B and C under the action of $\bar{\sigma}=100 \mathrm{MPa}$ (the amplitude for fully reversed cycles is then $200 \mathrm{MPa})$. 


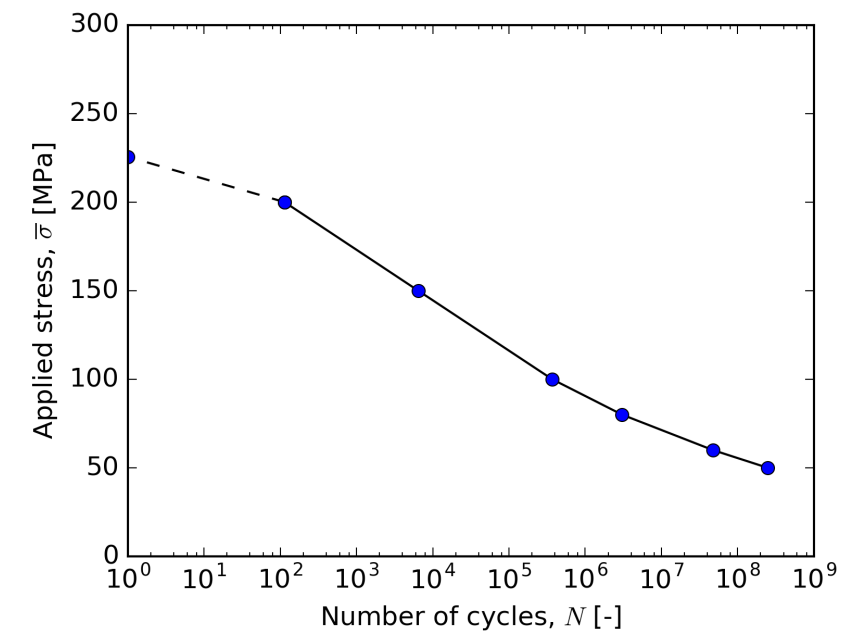

Figure 13: $\bar{\sigma}-N$ curve for the analysed fully 3D polycrystalline morphology A. benchmarking tool for other numerical techniques. As observed by McDowell and Dunne [13], the development of methods for the analysis of materials at the microstructural level is assuming increasing importance as it allows the assessment of the influence of features such as grain size, grain-size distribution, grain-boundary 560

bulk grains in a crystal plasticity context [44, 45, 52]. An interesting development could be addressed at the formulation of an extended tool for analysis of materials operating under the action of cyclic loads in aggressive environments [104]. Moreover, for the effective study of damage nucleation at the macro-scale, the developed microstructural model could be embedded within a concurrent multi-scale computational framework [61 62], which would allow linking the nucleation and evolution of material damage at the grain-scale with the failure of engineering components under the action of cyclic loads. Due to the likely high computational costs, such developments would greatly benefit from suitable computational acceleration schemes, within the context of the boundary element method [105, 106].

Within the limits of its assumptions, the developed method provides a crossquality, impurity segregation, etc. on the initiation and evolution of the damaging processes up to the transition of damage to the application macro-scale. Since the mentioned microscopic features are often directly related to the manufacturing pro- 
cesses, microstructural analyses may inform potential production routes for the attainment of optimal materials in terms of selected macro-properties, e.g. fatigue life. Additionally, models at the micro-scale can find direct applications in the analysis and design of MEMS, today routinely used in the fabrication of sensors, actuators, linkages, etc.

The predictive capabilities of similar models would greatly benefit from enhanced experimental material characterisation at the micro-scale, with enhanced assessment of those micro and nano-features that directly affect the behaviour of bulk grains and interfaces. For such purposes, also the employment of computational modelling at even lower scales, such as molecular mechanics, is a valuable tool, as it would support improved material characterisation of crystal lattices and grain boundaries, including impurity segregation.

575 However, the ability of perfectly simulating the behaviour of a specific aggregate should not be considered as the ultimate goal of similar formulations. Such target, if ever attainable, would be of limited interest with respect to the ability of inferring statistical information on the influence of selected micro-features on the aggregate mechanics, in which lies the strength of these microstructural models.

\section{Conclusions}

A grain-scale formulation for high-cycle fatigue degradation in polycrystalline aggregates has been proposed in this study. The model is based on a boundary integral representation of the aggregate micro-mechanics. The intergranular behaviour is modelled through cohesive interfaces whose high-cycle degradation is represented by continuum damage mechanics laws. High-cycle simulations are performed resorting to both an envelope-load and cycle-jump strategies. The method has been used for simulating the progressive degradation of microstructural polycrystalline components under cyclic loads and the obtained results are qualitatively sound and in line with those obtained in similar studies. The developed formulation is an alternative numerical tool for the study of fatigue damage and crack initiation in polycrystalline materials and MEMS. 


\section{Acknowledgements}

The authors acknowledge the support of CINECA's Italian Centre for Super Computing Applications and Innovation (SCAI) for access to and support in the use of their HPC infrastructure (http://www.hpc.cineca.it).

\section{Appendix A. Anisotropic Green's functions}

The kernels $U_{i j}^{g}(\boldsymbol{\xi}, \boldsymbol{\eta})$ and $T_{i j}^{g}(\boldsymbol{\xi}, \boldsymbol{\eta})$ in Eq.5) are given by

$$
U_{i j}^{g}(\boldsymbol{\xi}, \boldsymbol{\eta})=G_{i j}^{g}(\boldsymbol{\xi}, \boldsymbol{\eta}), \quad T_{i j}^{g}(\boldsymbol{\xi}, \boldsymbol{\eta})=n_{k}(\boldsymbol{\eta}) c_{j k p q} \frac{\partial G_{i p}^{g}}{\partial \eta_{q}}(\boldsymbol{\xi}, \boldsymbol{\eta})
$$

where $i, j, k, p, q=1,2,3, \boldsymbol{n}(\boldsymbol{\eta})=\left\{n_{i}(\boldsymbol{\eta})\right\}$ is the unit outward vector normal to the boundary at the point $\boldsymbol{\eta}$ and $G_{i j}^{g}(\boldsymbol{\xi}, \boldsymbol{\eta})$ are the 3D anisotropic Green's functions for the grain $g$, obtained as the solution of the differential system

$$
c_{i k j l} \frac{\partial^{2} G_{p j}^{g}}{\partial \eta_{k} \partial \eta_{l}}(\boldsymbol{\xi}, \boldsymbol{\eta})+\delta_{p i} \cdot \delta(\boldsymbol{\eta}-\boldsymbol{\xi})=0
$$

where $\boldsymbol{\xi}$ and $\boldsymbol{\eta}$ are the collocation and observation points, respectively, $c_{i j k l}$ is the anisotropic fourth-order elasticity tensor, $\delta_{p i}$ is the Kronecker delta and $\delta(\boldsymbol{\eta}-\boldsymbol{\xi})$ is the Dirac delta function. Using the Fourier transform with respect to $\boldsymbol{\eta}$ to Eq.A.2 and following in Ref.[77], the Green's functions $G_{i j}^{g}(\boldsymbol{\xi}, \boldsymbol{\eta})$ and their derivatives can be obtained in terms of spherical harmonics as follows

$$
\frac{\partial^{(I)} G_{i j}^{g}}{\partial r_{1}^{\left(\alpha_{1}\right)} \partial r_{2}^{\left(\alpha_{2}\right)} \partial r_{3}^{\left(\alpha_{3}\right)}}(\boldsymbol{r})=\frac{1}{4 \pi r^{I+1}} \sum_{\ell \in \mathcal{L}}^{\infty} P_{\ell}^{I}(0) \sum_{m=-\ell}^{\ell} \widetilde{G}_{i j,\left(\alpha_{1}, \alpha_{2}, \alpha_{3}\right)}^{\ell, m} Y_{\ell}^{m}(\hat{\boldsymbol{r}}),
$$

where $\boldsymbol{r} \equiv \boldsymbol{\eta}-\boldsymbol{\xi}, \boldsymbol{r}=\sqrt{r_{k} r_{k}}, \hat{\boldsymbol{r}}=\boldsymbol{r} / r ; I=\alpha_{1}+\alpha_{2}+\alpha_{3}$ denotes the order of derivation and $\mathcal{L}$ is the set of positive even (odd) integers when $I$ is even (odd). $P_{\ell}^{I}(0)$ is the $\ell$-th associated Legendre polynomials of degree $I$ evaluated at 0 and $Y_{\ell}^{m}(\hat{\boldsymbol{r}})$ is the spherical harmonic of order $\ell$ and degree $m$. The coefficients $\widetilde{G}_{i j,\left(\alpha_{1}, \alpha_{2}, \alpha_{3}\right)}^{\ell, m}$ of the series are computed by means of the following integral over the unit sphere $S_{1}$ :

$$
\widetilde{G}_{i j,\left(\alpha_{1}, \alpha_{2}, \alpha_{3}\right)}^{\ell, m}=\int_{S_{1}}\left(\hat{\xi}_{1}\right)^{\alpha_{1}}\left(\hat{\xi}_{2}\right)^{\alpha_{2}}\left(\hat{\xi}_{3}\right)^{\alpha_{3}} \widetilde{G}_{i j}(\hat{\boldsymbol{\xi}}) \bar{Y}_{\ell}^{m}(\hat{\boldsymbol{\xi}}) \mathrm{d} S(\hat{\boldsymbol{\xi}}),
$$

being $\widetilde{G}_{i j}(\boldsymbol{\xi})=\left[c_{i k j l} \xi_{k} \xi_{l}\right]^{-1}$ and $\bar{Y}_{\ell}^{m}$ the complex conjugate of $Y_{\ell}^{m}$. The interested readers are referred to Ref.[77] for further details. 


\section{References}

600 [1] S. Suresh, Fatigue of Materials, 2nd Edition, Cambridge University Press, 1998. doi:10.1017/CBO9780511806575

[2] J. Schijve, Fatigue of structures and materials, Springer Netherlands, 2001.

[3] C. Bathias, et al., Fatigue of materials and structures, John Wiley \& Sons, 2013.

[4] T. L. Anderson, Fracture mechanics: fundamentals and applications, CRC press, 2017.

[5] B. L. Bak, C. Sarrado, A. Turon, J. Costa, Delamination under fatigue loads in composite laminates: a review on the observed phenomenology and computational methods, Applied Mechanics Reviews 66 (6) (2014) 060803.

[6] A. Wöhler, Über die Festigkeits-versuche mit Eisen und Stahl, 1870.

[7] O. Basquin, The exponential law of endurance tests, in: Proc Astm, Vol. 10, 1910, pp. 625-630.

[8] T. Nicholas, J. Zuiker, On the use of the goodman diagram for high cycle fatigue design, International Journal of Fracture 80 (2-3) (1996) 219-235.

[9] J. Lemaitre, A course on damage mechanics, Springer, 1992.

[10] P. C. Paris, M. P. Gomez, W. E. Anderson, A rational analytic theory of fatigue, The trend in engineering 13 (1) (1961) 9-14.

[11] P. Paris, F. Erdogan, A critical analysis of crack propagation laws, ASME. Journal of Basic Engineering 85 (4) (1963) 528-533. doi:doi:10.1115/1. 3656900

${ }_{620}^{\text {口 }}$ [12] D. L. Davidson, J. Lankford, Fatigue crack growth in metals and alloys: mechanisms and micromechanics International Materials Reviews 37 (1) (1992) 4576. arXiv:https://doi.org/10.1179/imr.1992.37.1.45 doi:10.1179/imr.1992.37.1.45 URL https : //doi.org/10.1179/imr.1992.37.1.45 
${ }_{625}[13]$ D. McDowell, F. Dunne, Microstructure-sensitive computational modeling of fatigue crack formation. International Journal of Fatigue 32 (9) (2010) 1521 - 1542, emerging Frontiers in Fatigue. doi:https: //doi.org/10.1016/j.ijfatigue.2010.01.003

ㅁ URL http://wWw.sciencedirect.com/science/article/ $\mathrm{pii/S0142112310000162}$

[14] K. S. Chan, Roles of microstructure in fatigue crack initiation International Journal of Fatigue 32 (9) (2010) 1428 - 1447, emerging Frontiers in Fatigue.

doi:https://doi.org/10.1016/j.ijfatigue.2009.10. 005 .

${ }_{635}$ URL http://WWW.sciencedirect.com/science/article/ pii/S0142112309002989

[15] S. Subramanian, K. Reifsnider, W. Stinchcomb, A cumulative damage model

घ to predict the fatigue life of composite laminates including the effect of a fibre-matrix interphase International Journal of Fatigue 17 (5) (1995) 343 - 351.

${ }_{640}$ doi:https://doi.org/10.1016/0142-1123(95)99735-S

口 URL http://wWW.sciencedirect.com/science/article/ pii/014211239599735S

口 [16] N. V. Akshantala, R. Talreja, A micromechanics based model for predicting fatigue life of composite laminates Materials Science and Engineering: A 285 (1) (2000) 303 - 313. doi:https://doi.org/10.1016/ S0921-5093(00)00679-1. URL http://wWw.sciencedirect.com/science/article/ pii/s0921509300006791

[17] J. Degrieck, W. Van Paepegem, Fatigue damage modeling of fibre-reinforced composite materials, Applied mechanics reviews 54 (4) (2001) 279-300.

[18] K. Saanouni, A. Abdul-Latif, Micromechanical modeling of low cy口 cle fatigue under complex loadings fi!? part i. theoretical formulation International Journal of Plasticity 12 (9) (1996) 1111 - 1121. 
doi:https://doi.org/10.1016/S0749-6419(96)00043-5

URL http://www.sciencedirect.com/science/article/ pii/S0749641996000435

[19] T. Watanabe, S. Tsurekawa, The control of brittleness and development of desirable mechanical properties in polycrystalline systems by grain boundary engineering, Acta Materialia 47 (15) (1999) 4171 - 4185. doi:https://doi.org/10.1016/S1359-6454(99)00275-X URL http://WWW.sciencedirect.com/science/article/ pii/S135964549900275X

[ [20] J. Banhart, Manufacture, characterisation and application of cellular metals

1 and metal foams Progress in Materials Science 46 (6) (2001) 559 - 632. doi :

665 http://dx.doi.org/10.1016/S0079-6425(00)00002-5

a URL http://wWw.sciencedirect.com/science/article/ pii/s0079642500000025

[ [21] M. Rashed, M. Ashraf, R. Mines, P. J. Hazell, Metallic microlattice mate-

1. rials: A current state of the art on manufacturing, mechanical properties

670 and applications Materials \& Design 95 (Supplement C) (2016) 518 - 533. doi:https://doi.org/10.1016/j.matdes.2016.01.146

口 URL http://www.sciencedirect.com/science/article/ pii/S0264127516301447

口 [22] D. J. Bell, T. J. Lu, N. A. Fleck, S. M. Spearing, Mems actuators and sensors: observations on their performance and selection for purpose. Journal of Micromechanics and Microengineering 15 (7) (2005) S153.

URL http://stacks . iop.org/0960-1317/15/i=7/a=022

[23] A. King, G. Johnson, D. Engelberg, W. Ludwig, J. Marrow, Observations of intergranular stress corrosion cracking in a grain-mapped polycrystal, Science 321 (5887) (2008) 382-385.

[24] W. Ludwig, A. King, P. Reischig, M. Herbig, E. Lauridsen, S. Schmidt, H. Proudhon, S. Forest, P. Cloetens, S. R. du Roscoat, J. Buffire, T. Marrow, H. Poulsen, 
New opportunities for 3d materials science of polycrystalline materials at
the micrometre lengthscale by combined use of x-ray diffraction and x-ray
imaging Materials Science and Engineering: A 524 (1) (2009) $69-76$, special
Topic Section: Probing strains and Dislocation Gradients with diffraction.
doi:https://doi.org/10.1016/j.msea.2009.04.009.
URL http://www.sciencedirect.com/science/article/
pii/S0921509309004511

${ }_{690}$ [25] M. T. Lusk, A. E. Mattsson, High-performance computing for materials de-

1 sign to advance energy science, MRS Bulletin 36 (3) (2011) 169?174. doi: $10.1557 / \mathrm{mrs} .2011 .30$

¿ [26] F. Dunne, Fatigue crack nucleation: Mechanistic modelling across the length scales Current Opinion in Solid State and Materials Science 18 (4) (2014) 170 179, slip Localization and Transfer in Deformation and Fatigue of Polycrystals. doi:https://doi.org/10.1016/j.cossms.2014.02.005

a URL http://WWW.sciencedirect.com/science/article/ pii/S1359028614000151

[ [27] W. Spengen, R. Modlinski, R. Puers, A. Jourdain, Failure Mechanisms in MEMS/NEMS Devices Springer Berlin Heidelberg, Berlin, Heidelberg, 2007, pp. 1663-1684. doi:10 . 1007/978-3-540-29857-1_52 URL https : //doi.org/10 . 1007/978-3-540-29857-1_52

[28] B. Jalalahmadi, F. Sadeghi, D. Peroulis, A numerical fatigue damage model for life scatter of mems devices, Journal of Microelectromechanical Systems 18 (5) (2009) 1016-1031.

¿ [29] A. Warhadpande, B. Jalalahmadi, T. Slack, F. Sadeghi, A new finite element fatigue modeling approach for life scatter in tensile steel specimens International Journal of Fatigue 32 (4) (2010) 685 - 697. doi:https: //doi.org/10.1016/j.ijfatigue.2009.10.003

710 uRL http://WWW.sciencedirect.com/science/article/ pii/S0142112309002965 
[ [30] G. M. Castelluccio, W. D. Musinski, D. L. McDowell, Recent developments in assessing microstructure-sensitive early stage fatigue of polycrystals Current Opinion in Solid State and Materials Science 18 (4) (2014) 180 - 187, slip Localization and Transfer in Deformation and Fatigue of Polycrystals. doi:https://doi.org/10.1016/j.cossms.2014.03.001 URL http://wWw.sciencedirect.com/science/article/ pii/S1359028614000163

[ [31] H. Kahn, N. Tayebi, R. Ballarini, R. Mullen, A. Heuer, Fracture toughness of polysilicon mems devices Sensors and Actuators A: Physical 82 (1) (2000) 274 - 280. doi:https://doi.org/10.1016/S0924-4247(99) $00366-0$

प URL http://wWw.sciencedirect.com/science/article/ pii/S0924424799003660

${ }_{725}$ [32] W. Sharpe, J. Bagdahn, Fatigue testing of polysiliconfi?! - fi?!a review, Mechanics of Materials 36 (1) (2004) 3 - 11, fatigue of Advanced Materials. doi:https://doi.org/10.1016/S0167-6636(03)00027-9

【 URL http://wWw.sciencedirect.com/science/article/ pii/s0167663603000279

730 [33] H. Kahn, L. Chen, R. Ballarini, A. Heuer, Mechanical fatigue of polysilicon: Effects of mean stress and stress amplitude. Acta Materialia 54 (3) (2006) 667 - 678. doi:https://doi.org/10.1016/j.actamat.2005.10.007

口 URL http://www.sciencedirect.com/science/article/ pii/S1359645405005902

${ }_{735}$ [34] F. Roters, P. Eisenlohr, L. Hantcherli, D. D. Tjahjanto, T. R. Bieler, D. Raabe, Overview of constitutive laws, kinematics, homogenization and multiscale methods in crystal plasticity finite-element modeling: Theory, experiments, applications, Acta Materialia 58 (4) (2010) 1152-1211.

[35] I. Benedetti, F. Barbe, Modelling polycrystalline materials: an overview of 
three-dimensional grain-scale mechanical models, Journal of Multiscale Modelling 5 (01) (2013) 1350002.

[36] F. Barbe, L. Decker, D. Jeulin, G. Cailletaud, Intergranular and intragranular behavior of polycrystalline aggregates. part 1: F.e. model International Journal of Plasticity 17 (4) (2001) 513 - 536. doi:https: //doi.org/10.1016/S0749-6419(00)00061-9

口 URL http://www.sciencedirect.com/science/article/ pii/s0749641900000619

¿ [37] F. Fritzen, T. Böhlke, E. Schnack, Periodic three-dimensional mesh generation for crystalline aggregates based on voronoi tessellations Computational Mechanics 43 (5) (2009) 701-713. doi : 10 . 1007/s00466-008-0339-2 URL https://doi.org/10.1007/s00466-008-0339-2

[38] H. D. Espinosa, P. D. Zavattieri, A grain level model for the study of failure initiation and evolution in polycrystalline brittle materials. part i: Theory and numerical implementation, Mechanics of Materials 35 (3) (2003) 333-364.

[39] H. D. Espinosa, P. D. Zavattieri, A grain level model for the study of failure initiation and evolution in polycrystalline brittle materials. part ii: numerical examples, Mechanics of Materials 35 (3) (2003) 365-394.

[40] A. Musienko, G. Cailletaud, Simulation of inter- and transgranular crack propagation in polycrystalline aggregates due to stress corrosion cracking, Acta Materialia 57 (13) (2009) 3840-3855. doi : 10 . 1016/j . actamat . 2009 . 04.035

[41] M. Kamaya, M. Itakura, Simulation for intergranular stress corrosion cracking based on a three-dimensional polycrystalline model, Engineering Fracture Mechanics 76 (3) (2009) $386-401$.

[42] J. Rimoli, M. Ortiz, A three-dimensional multiscale model of intergranular hydrogen-assisted cracking, Philosophical Magazine 90 (21) (2010) 2939-2963. 
[43] I. Simonovski, L. Cizelj, Towards modeling intergranular stress corrosion cracks on grain size scales, Nuclear Engineering and Design 246 (0) (2012) 107-114.

[ [44] A. Manonukul, F. P. E. Dunne, High- and low-cycle fatigue crack initiation

[45] K.-S. Cheong, M. J. Smillie, D. M. Knowles, Predicting fatigue crack initiation through image-based micromechanical modeling Acta Materialia 55 (5) (2007)

[46] C. Przybyla, R. Prasannavenkatesan, N. Salajegheh, D. L. McDowell, Microstructure-sensitive modeling of high cycle fatigue Inter-

[47] J. Bomidi, N. Weinzapfel, F. Sadeghi, Three-dimensional modelling of intergranular fatigue failure of fine grain polycrystalline metallic mems devices, Fatigue \& Fracture of Engineering Materials \& Structures 35 (11) (2012) 1007-1021.

[ [48] O. Kraft, R. Schwaiger, P. Wellner, Fatigue in thin films: lifetime and damage formation Materials Science and Engineering: A 319-321 (Supplement C) (2001) $795 \quad \square$ 919 - 923. doi:https://doi.org/10.1016/S0921-5093(01) 
$00990-\mathrm{X}$

URL http://www.sciencedirect.com/science/article/ pii/S092150930100990X

口 [49] J. Aktaa, J. Reszat, M. Walter, K. Bade, K. Hemker, High cycle fatigue and frac-

800 ture behavior of liga nickel Scripta Materialia 52 (12) (2005) $1217-1221$. do i : https://doi.org/10.1016/j.scriptamat.2005.03.004

口 URL http://www.sciencedirect.com/science/article/ pii/S1359646205001296

[50] G. Zhang, C. Volkert, R. Schwaiger, P. Wellner, E. Arzt, O. Kraft, Length-

805 scale-controlled fatigue mechanisms in thin copper films Acta Materialia 54 (11) (2006) 3127 - 3139. doi:https://doi.org/10.1016/j . actamat.2006.03.013 URL http://www.sciencedirect.com/science/article/ pii/S1359645406002023

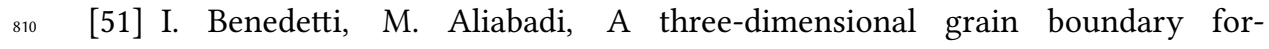
mulation for microstructural modeling of polycrystalline materials

n Computational Materials Science 67 (2013) 249 - 260. doi:http: //dx.doi.org/10.1016/j.commatsci.2012.08.006

a URL //WWW.sciencedirect.com/science/article/pii/ ${ }_{815} \quad$ S0927025612004958

„ [52] I. Benedetti, V. Gulizzi, V. Mallardo, A grain boundary formulation for crystal

10 plasticity. International Journal of Plasticity 83 (2016) $202-224$. doi : http : //dx.doi.org/10.1016/j.ijplas.2016.04.010

I) URL //WWW.sciencedirect.com/science/article/pii/ S0749641916300596

[53] G. Sfantos, M. Aliabadi, A boundary cohesive grain element formulation for modelling intergranular microfracture in polycrystalline brittle materials, International journal for numerical methods in engineering 69 (8) (2007) 15901626. doi: $10.1002 / \mathrm{nme} .1831$ 
[54] I. Benedetti, M. Aliabadi, A three-dimensional cohesive-frictional grainboundary micromechanical model for intergranular degradation and failure in polycrystalline materials, Computer Methods in Applied Mechanics and Engineering 265 (2013) 36-62. doi : 10 . 1016/j . cma.2013.05.023

[55] V. Gulizzi, A. Milazzo, I. Benedetti, An enhanced grain-boundary framework for computational homogenization and micro-cracking simulations of polycrystalline materials, Computational Mechanics 56 (4) (2015) 631-651. doi : 10.1007/s00466-015-1192-8

[56] V. Gulizzi, I. Benedetti, Micro-cracking of brittle polycrystalline materials with initial damage, European Journal of Computational Mechanics 25 (1-2) (2016) $38-53$.

[ [57] V. Gulizzi, C. Rycroft, I. Benedetti, Modelling intergranular and transgranular micro-cracking in polycrystalline materials Computer Methods in Applied Mechanics and Engineering (2017)-doi:https://doi.org/10.1016/ j.cma.2017.10.005

URL https://www.sciencedirect.com/science/article/ pii/S0045782517306746

[58] G. Geraci, M. Aliabadi, Micromechanical boundary element modelling of transgranular and intergranular cohesive cracking in polycrystalline materials, Engineering Fracture Mechanics 176 (2017) 351-374.

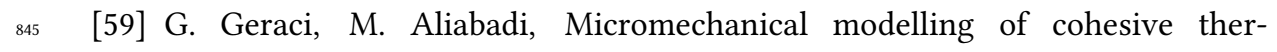
moelastic cracking in orthotropic polycrystalline materials Computer Methods in Applied Mechanics and Engineering 339 (2018) 567 - 590. doi:https://doi.org/10.1016/j.cma.2018.05.011 URL http://WWW.sciencedirect.com/science/article/ 850 pii/S0045782518302548

[ [60] I. Benedetti, V. Gulizzi, A. Milazzo, Grain-boundary modelling of hydrogen assisted intergranular stress corrosion cracking Mechanics of Materials 117 
(2018) 137-151. doi:10.1016/j.mechmat.2017.11.001

$\begin{array}{ll}\text { प URL } & \text { https://WwW.scopus.com/inward/record. } \\ \text { uri?eid=2-s2.0-85037155275\&doi=10.1016\% } \\ \text { 2fj.mechmat.2017.11.001\&partnerID=40\&md5= } \\ \text { 38ef17b25776893b9871f8378ae3d731 }\end{array}$

[61] G. Sfantos, M. Aliabadi, Multi-scale boundary element modelling of material degradation and fracture, Computer Methods in Applied Mechanics and En-

${ }_{860}$ a gineering 196 (7) (2007) 1310-1329. doi:10.1016/j.cma.2006.09. 004

[62] I. Benedetti, M. Aliabadi, Multiscale modeling of polycrystalline materials: A boundary element approach to material degradation and fracture, Computer Methods in Applied Mechanics and Engineering 289 (2015) 429 - 453. doi : http://dx.doi.org/10.1016/j.cma.2015.02.018

[63] M. H. Aliabadi, The boundary element method: applications in solids and structures., Vol. 2, John Wiley \& Sons Ltd, England, 2002.

q [64] A. Jivkov, N. Stevens, T. Marrow, A three-dimensional computational model for intergranular cracking, Computational Materials Science 38 (2) (2006) 442

${ }_{870}$ 口 - 453. doi:https://doi.org/10.1016/j.commatsci.2006. 03.012

口 URL http://wWw.sciencedirect.com/science/article/ pii/s0927025606000863

[ [65] I. Simonovski, L. Cizelj, Automatic parallel generation of finite element meshes ${ }_{875}$ for complex spatial structures Computational Materials Science 50 (5) (2011)

a 1606 - 1618. doi:https://doi.org/10.1016/j.commatsci. 2010.12 .014

a URL http://WWW.sciencedirect.com/science/article/ pii/s0927025610006713

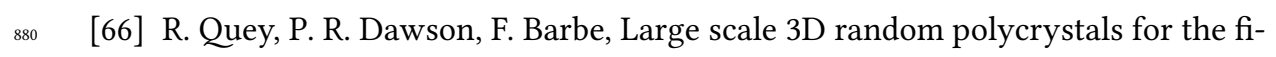


nite element method: Generation, meshing and remeshing, Computer Methods in Applied Mechanics and Engineering 200 (2011) 1729-1745.

[67] C. Lautensack, S. Zuyev, Random laguerre tessellations, Advances in Applied Probability 40 (3) (2008) 630-650. doi:10.1017/ S000186780000272X

[68] A. Lyckegaard, E. M. Lauridsen, W. Ludwig, R. W. Fonda, H. F. Poulsen, On the use of laguerre tessellations for representations of $3 \mathrm{~d}$ grain structures Advanced Engineering Materials 13 (3) (2011) 165-170. doi : 10 . 1002/adem. 201000258

${ }_{890}$ URL http://dx.doi.org/10.1002/adem.201000258

口 [69] R. Quey, L. Renversade, Optimal polyhedral description of 3d polya crystals: Method and application to statistical and synchrotron $\mathrm{x}$ ray diffraction data Computer Methods in Applied Mechanics and Engineering 330 (Supplement C) (2018) 308 - 333.

doi:https: //doi.org/10.1016/j.cma.2017.10.029 URL http://www.sciencedirect.com/science/article/ pii/s0045782517307028

[70] C. H. Rycroft, Voro++: A three-dimensional Voronoi cell library in $\mathrm{C}++$, Chaos 19 (2009) 041111.

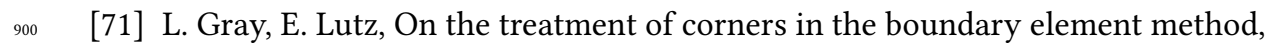
Journal of Computational and Applied Mathematics 32 (3) (1990) 369 - 386. doi:https://doi.org/10.1016/0377-0427(90)90043-Y

1 URL http://WwW.sciencedirect.com/science/article/ pii/037704279090043Y

${ }_{905}^{\text {口 }}$ [72] Q. Deng, C. G. Li, S. L. Wang, H. Tang, H. Zheng, A new method to the treatment of corners in the bem Engineering Analysis with Boundary Elements 37 (1) (2013) 182 - 186. doi:https://doi.org/10.1016/j. enganabound.2012.03.017 
URL http://wWw.sciencedirect.com/science/article/ pii/S0955799712001439

[73] J. R. Rice, Mathematical analysis in the mechanics of fracture, Fracture: an advanced treatise 2 (1968) 191-311.

[74] H. D. Espinosa, P. D. Zavattieri, A grain level model for the study of failure initiation and evolution in polycrystalline brittle materials. part ii: numerical examples, Mechanics of Materials 35 (3) (2003) 365-394.

[75] V. Tomar, J. Zhai, M. Zhou, Bounds for element size in a variable stiffness cohesive finite element model, International journal for numerical methods in engineering 61 (11) (2004) 1894-1920.

[76] P. Banerjee, The boundary element methods in engineering, McGraw-Hill, 1994, ${ }_{920} \quad$ (pp. 177-188).

[77] V. Gulizzi, A. Milazzo, I. Benedetti, Fundamental solutions for general anisotropic multi-field materials based on spherical harmonics expansions, International Journal of Solids and Structures 100 (2016) 169-186. doi:10. $1016 / j$.ijsolstr.2016.08.014

${ }_{925}^{\text {口 }}$ [78] V. Deshpande, A. Needleman, E. V. der Giessen, Scaling of discrete dislocation predictions for near-threshold fatigue crack growth Acta Materialia 51 (15) (2003) 4637 - 4651. doi:https://doi.org/10.1016/ S1359-6454(03)00302-1. URL http://wWw.sciencedirect.com/science/article/ pii/S1359645403003021

[79] S. Groh, S. Olarnrithinun, W. Curtin, A. Needleman, V. Deshpande, E. V. der Giessen, Fatigue crack growth from a cracked elastic particle into a ductile matrix Philosophical Magazine 88 (30-32) (2008) 3565-3583. arXiv:http://dx.doi.org/10.1080/14786430802320127 doi:10.1080/14786430802320127 URL http://dx .doi.org/10.1080/14786430802320127 
口 [80] O. Nguyen, E. Repetto, M. Ortiz, R. Radovitzky, A cohesive model of fatigue crack growth International Journal of Fracture 110 (4) (2001) 351-369. doi : 10.1023/A: 1010839522926 URL https://doi.org/10.1023/A:1010839522926

[ [81] K. Roe, T. Siegmund, An irreversible cohesive zone model for interface fatigue crack growth simulation Engineering Fracture Mechanics 70 (2) (2003) 209

口 - 232. doi:https://doi.org/10.1016/S0013-7944(02) $00034-6$

${ }_{945}^{\square}$ URL http://WWW.sciencedirect.com/science/article/ pii/s0013794402000346

[82] R. H. J. Peerlings, W. A. M. Brekelmans, R. de Borst, M. G. D. Geers, Gradient-enhanced damage modelling of high-cycle fatigue International Journal for Numerical Methods in Engineering 49 (12) (2000) 1547-

950 1 1569. doi:10.1002/1097-0207(20001230)49:12〈1547:: AID-NME16>3.0.CO;2-D

10 URL http://dx.doi.org/10.1002/1097-0207(20001230) $49: 12\langle 1547::$ AID-NME16〉3.0.CO;2-D

口 [83] P. Robinson, U. Galvanetto, D. Tumino, G. Bellucci, D. Violeau, Numerical simulation of fatigue-driven delamination using interface elements. International Journal for Numerical Methods in Engineering 63 (13) (2005) 1824-1848. doi:10.1002/nme.1338 URL http://dx.doi.org/10.1002/nme.1338

[ [84] J. Muoz, U. Galvanetto, P. Robinson, On the numerical simulation of fatigue driven delamination with interface elements International Journal of Fatigue 28 (10) (2006) 1136 - 1146, the Third International Conference on Fatigue of

a Composites. doi:https://doi.org/10.1016/j.ijfatigue. 2006.02 .003

1 URL http://wWw.sciencedirect.com/science/article/ ${ }_{965} \quad \mathrm{pii} / \mathrm{S} 0142112306000338$ 
[85] D. Tumino, F. Cappello, Simulation of fatigue delamination growth in composites with different mode mixtures Journal of Composite Materials 41 (20) (2007) 2415-2441. arXiv: https://doi.org/10 .1177/ 0021998307075439 doi:10.1177/0021998307075439. URL https://doi.org/10.1177/0021998307075439

[86] A. Turon, J. Costa, P. Camanho, C. Dvila, Simulation of delamination in composites under high-cycle fatigue Composites Part A: Applied Science and Manufacturing 38 (11) (2007) 2270 - 2282, compTest 2006. doi:https: //doi.org/10.1016/j.compositesa.2006.11.009 URL http://www.sciencedirect.com/science/article/ pii/S1359835X0600337X

[ [87] L. F. Kawashita, S. R. Hallett, A crack tip tracking algorithm for cohesive

i interface element analysis of fatigue delamination propagation in composite materials International Journal of Solids and Structures 49 (21) (2012) 2898

$980 \quad-2913$. doi:https://doi.org/10.1016/j.ijsolstr.2012. 03.034

I) URL http://www.sciencedirect.com/science/article/ pii/S0020768312001369

[88] B. L. V. Bak, A. Turon, E. Lindgaard, E. Lund, A simulation method for high-cycle fatigue-driven delamination using a cohesive zone model International Journal for Numerical Methods in Engineering 106 (3) (2016) 163-191, nme.5117. doi:10.1002/nme.5117 URL http : //dx .doi.org/10 .1002/nme.5117

[89] L. M. KACHANOV, Time of the rupture process under creep conditions, izy akad, Nank SSR Otd Tech Nauk 8 (1958) 26-31.

[90] Y. Robotnov, Creep problems in structural mechanics (1969).

[91] J. L. Chaboche, P. M. Lesne, A non-linear continuous fatigue damage model Fatigue \& Fracture of Engineering Materials \& Structures 11 (1) (1988) 1-17. 


$$
\begin{aligned}
& \text { doi:10.1111/j.1460-2695.1988.tb01216.x } \\
& \text { URL http://dx.doi.org/10.1111/j.1460-2695.1988. } \\
& \text { tb01216.x } \\
& \text { [92] Y.-C. Xiao, S. Li, Z. Gao, A continuum damage mechanics model for high } \\
& \text { cycle fatigue International Journal of Fatigue } 20 \text { (7) (1998) } 503-508 . \\
& \text { doi:https://doi.org/10.1016/S0142-1123(98)00005-X } \\
& \text { URL http://WWw.sciencedirect.com/science/article/ } \\
& \text { pii/S014211239800005X }
\end{aligned}
$$

[93] V. V. Bolotin, Mechanics of fatigue, Vol. 11, CRC Press, 1999.

[ [94] W. V. Paepegem, J. Degrieck, Fatigue degradation modelling of plain woven glass/epoxy composites Composites Part A: Applied Sci-

1005 ence and Manufacturing 32 (10) (2001) 1433 - 1441. doi:https: //doi.org/10.1016/S1359-835X(01)00042-2

a URL http://WWW.sciencedirect.com/science/article/ pii/S1359835X01000422

[95] A. Kuzmin, M. Luisier, O. Schenk, Fast methods for computing selected elements of the greens function in massively parallel nanoelectronic device simulations, in: F. Wolf, B. Mohr, D. Mey (Eds.), Euro-Par 2013 Parallel Processing, Vol. 8097 of Lecture Notes in Computer Science, Springer Berlin Heidelberg, 2013, pp. 533-544.

[96] O. Schenk, M. Bollhöfer, R. A. Römer, On large-scale diagonalization techniques for the anderson model of localization, SIAM Rev. 50 (1) (2008) 91-112.

[97] O. Schenk, A. Wttchter, M. Hagemann, Matching-based preprocessing algorithms to the solution of saddle-point problems in large-scale nonconvex interior-point optimization, Computational Optimization and Applications $36(2-3)(2007) 321-341$.

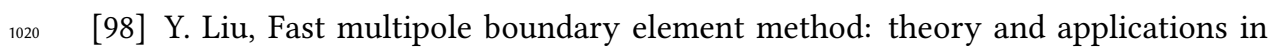
engineering, Cambridge university press, 2009. 
[99] M. Bebendorf, Hierarchical matrices: a means to efficiently solve elliptic boundary value problems, Vol. 63, Springer Science \& Business Media, 2008.

[100] I. Benedetti, M. Aliabadi, G. Davì, A fast 3d dual boundary ele-

口

[104] R. N. Parkins, B. S. Greenwell, The interface between corrosion fa-

[102] T. Buchheit, B. Boyce, G. Wellman, The role of microstructure in mems deformation and failure, in: Proc. IMECE, 2002, pp. 559-566.

[103] Z. Hashin, S. Shtrikman, A variational approach to the theory of the elastic behaviour of polycrystals, Journal of the Mechanics and Physics of Solids 10 (4) (1962) 343-352. tigue and stress-corrosion cracking Metal Science 11 (8-9) (1977) 405-

1 413. arXiv:https://doi.org/10.1179/msc.1977.11.8-9. 405 doi:10.1179/msc.1977.11.8-9.405

URL https://doi.org/10.1179/msc.1977.11.8-9.405

[105] I. Benedetti, M. H. Aliabadi, A fast hierarchical dual boundary element method for three-dimensional elastodynamic crack problems International Journal for

口 Numerical Methods in Engineering 84 (9) (2010) 1038-1067. doi : 10 . $1002 /$ nme.2929

URL http://dx.doi.org/10.1002/nme.2929 
[106] A. Milazzo, I. Benedetti, M. Aliabadi, Hierarchical fast bem for anisotropic time-harmonic 3-d elastodynamics Computers \& Structures 96-97 (Supplement C) (2012) 9 - 24. doi:https://doi.org/10.1016/j. compstruc.2012.01.010

a URL http://WWW.sciencedirect.com/science/article/ pii/S0045794912000259 